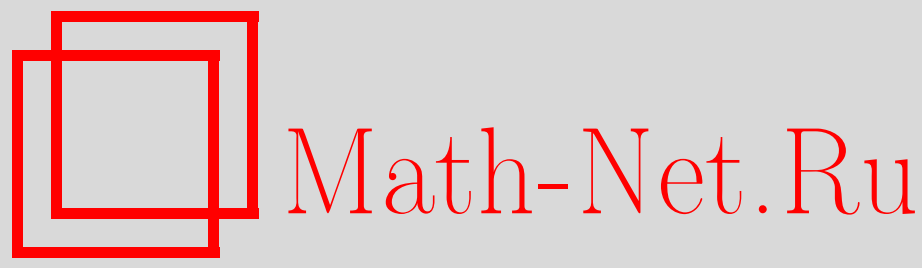

М. Д. Абловиц, Бао-Фэн Фэн, Сюй-Дань Ло, З. Мусслимани, Метод обратной задачи рассеяния для нелокального нелинейного уравнения Шредингера с обращением пространства-времени, ТМФ, 2018, том 196, номер 3, 343-372

DOI: https://doi.org/10.4213/tmf9449

Использование Общероссийского математического портала Math-Net.Ru подразумевает, что вы прочитали и согласны с пользовательским соглашением http: //www . mathnet.ru/rus/agreement

Параметры загрузки:

IP : 3.85 .73 .92

26 апреля 2023 г., 13:00:50

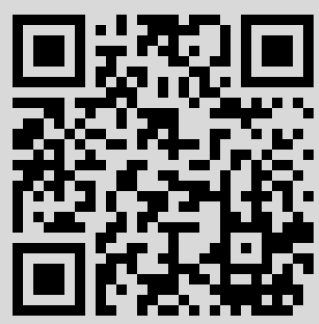




\title{
ФИЗИКА
}

Том 196, № 3

сентябрь, 2018

(C) 2018 г. М. Д. Абловиц*, Бао-Фэн Фэн ${ }^{\dagger}$, Сюй-Дань Ло 3. Мусслимани

\section{МЕТОД ОБРАТНОЙ ЗАДАЧИ РАССЕЯНИЯ ДЛЯ НЕЛОКАЛЬНОГО НЕЛИНЕЙНОГО УРАВНЕНИЯ ШРЕДИНГЕРА С ОБРАЩЕНИЕМ ПРОСТРАНСТВА-ВРЕМЕНИ}

\begin{abstract}
Недавно были введены нелокальные уравнения типа нелинейного уравнения Шредингера с обращением пространства-времени, а также было показано, что они являются интегрируемыми бесконечномерными динамическими системами, и построен метод обратной задачи рассеяния для быстроубывающих начальных условий. Здесь представлен метод обратной задачи рассеяния для нелинейного уравнения Шредингера с обращением времени с ненулевыми граничными условиями на бесконечности. Такая задача труднее из-за сложной структуры ветвления связанных с ней линейных собственных функций. Проанализированы два случая, соответствующие двум различным значениям фазы на бесконечности. Обсуждаются специальные солитонные решения и в явном виде построены одно- и двухсолитонные решения. Также рассмотрены пространственно-зависимые граничные условия.
\end{abstract}

Ключевые слова: обратная задача рассеяния, нелокальное нелинейное уравнение Шредингера с обращением пространства-времени.

DOI: https://doi.org/10.4213/tmf9449

Работа М.Д. Абловица частично поддержана NSF (грант № DMS-1310200), Бао-Фэн Фэн частично поддержан NSF (грант № DMS-171599) и NSFC for Overseas Scholar Collaboration Research (№ 11728103).

* Department of Applied Mathematics, University of Colorado at Boulder, Boulder, CO, USA. E-mail: mark.ablowitz@colorado.edu

${ }^{\dagger}$ School of Mathematical and Statistical Sciences, University of Texas Rio Grande Valley, Edinburg, TX, USA. E-mail: baofeng.feng@utrgv.edu

${ }^{\ddagger}$ Department of Mathematics, State University of New York at Buffalo, Buffalo, NY, USA. E-mail: xudanluo@buffalo.edu

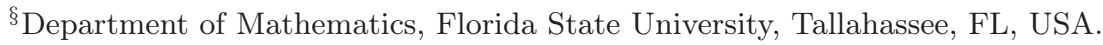

E-mail: muslimani@math.fsu.edu 


\section{1. ВВЕДЕНИЕ}

В 1965 г. Зубацкий и Крускал обнаружили [1], что решения уравнения Кортевега-де Фриза $(\mathrm{Kд} \Phi)$ в виде уединенных волн проявляют особые свойства при взаимодействии; они назвали эти решения солитонами. Это послужило мотивацией Гарднеру, Грину, Крускалу и Миуре [2] в 1967 г. связать уравнение КдФ с линейным уравнением Шредингера для быстроубывающих начальных условий на прямой и наметить подход к решению задачи Коши для уравнения КдФ с помощью метода обратной задачи рассеяния (МОЗР). Лакс узнал об этих результатах и вскоре показал [3], что уравнение КдФ и другие уравнения можно получить из условия совместности двух линейных операторов; одним из таких операторов для уравнения КдФ было линейное уравнение Шредингера.

В 1972 г. Захаров и Шабат обнаружили [4], что еще одно физически важное уравнение, нелинейное уравнение Шредингера (НУШ), также имеет пару Лакса и может быть решено (линеаризовано) с помощью МОЗР. Было известно, что НУШ, как и уравнение КдФ, возникает повсюду [5]. Под влиянием этих результатов в 1973 г. Абловиц, Кауп, Ньюэлл и Сегур (АКНС) обобщили [6] линейные операторы, использованные Захаровым и Шабатом; они показали, что НУШ, уравнение синус-Гордон [7], модифицированное уравнение КдФ, уравнение КдФ можно решить (линеаризовать) с помощью МОЗР. Вскоре после этого, в 1974 г. [8], был развит общий подход для нахождения интегрируемых систем, решаемых методом, который авторы назвали МОЗР. Метод был связан с классами уравнений (которые позже назвали рекуррентными операторами) и был применен для нахождения решений задачи с начальными условиями, быстроубывающими на прямой.

Чтобы решить нелинейное уравнение с помощью МОЗР, нужно сначала связать [9]-[11] нелинейное волновое уравнение с совместной линейной задачей рассеяния (или спектральной задачей) или парой Лакса, одно из уравнений которой представимо в виде $v_{x}=X v$, а соответствующее эволюционное уравнение - в виде $v_{t}=T v$. В операторе $X$ есть функция (или функции), называемая потенциалом (или потенциалами). Оператор $X$ и соответствующий линейный оператор эволюции во времени, обозначенный $T$, взаимно совместны с нелинейным волновым уравнением, которому удовлетворяет потенциал. Здесь мы обозначим потенциал (решение) нелинейного уравнения через $q(x, t)$.

В работе [8] было предложено рассмотреть задачу рассеяния

$$
v_{x}=X v=\left(\begin{array}{cc}
-i k & q(x, t) \\
r(x, t) & i k
\end{array}\right) v,
$$

где $v(x, t)=\left(v_{1}(x, t), v_{2}(x, t)\right)^{\mathrm{T}}, k$ - не зависящий от времени спектральный параметр, a $q(x, t), r(x, t)$ - комплексные функции вещественных переменных $x, t$. С задачей рассеяния АКНС (1.1) связано эволюционное уравнение

$$
v_{t}=T v,
$$

где $(2 \times 2)$-матрица $T$ является функцией от $q(x, t), r(x, t)$ и спектрального параметpa $k$. Различные матрицы $T$ порождают различные спаренные уравнения в частных производных для $q(x, t)$ и $r(x, t)$ из условия совместности $v_{x t}=v_{t x}$. При наличии 
определенных связей между $q(x, t)$ и $r(x, t)$ (которые называются также симметрийными редукциями) получается совместная система, которая сводится к одному интегрируемому эволюционному уравнению для $q(x, t)$ или $r(x, t)$. Важным примером является НУШ

$$
i q_{t}=q_{x x}-2 \sigma|q|^{2} q, \quad \sigma=\mp 1 .
$$

Многие исследования МОЗР посвящены задаче с быстроубывающими начальными условиями; например, $q(x, t), r(x, t) \rightarrow 0$ быстро при $x \rightarrow \pm \infty$ (см. работы [9]-[11]). Однако живой интерес был проявлен к другим задачам типа НУШ с ненулевыми граничными условиями. Первое исследование такого рода было проведено для НУШ [12]. В первоначальном МОЗР для ненулевых граничных условий использовались две поверхности Римана, связанные с точкой ветвления квадратного корня в собственных функциях и данных рассеяния. Важное продвижение было сделано с введением униформизующей переменной [13], что позволило привести обратную задачу к более стандартной обратной задаче на верхней или нижней полуплоскости новой переменной. Впоследствии многие ученые исследовали НУШ и связанные с ним задачи именно таким способом (см. [14]-[21]), таким образом существенно расширив применимость и диапазон использования МОЗР.

Недавно были найдены новые нелокальные симметрийные редукции в задаче рассеяния АКНС [22]. Среди них (здесь $\sigma=\mp 1)$ :

- $r(x, t)=\sigma q^{*}(x,-t)$;

- $r(x, t)=\sigma q^{*}(-x, t)$;

- $r(x, t)=\sigma q(-x,-t)$;

- $r(x, t)=\sigma q^{*}(-x,-t)$.

Каждая из этих симметрийных редукций приводит к новому классу нелокальных нелинейных интегрируемых уравнений и новому типу обратных задач, при этом была построена ОЗР с убывающими данными для многих соответствующих уравнений [22]. Важным примером является PT-симметричное НУШ [23], [24], нелокальное $P$ T-симметричное уравнение Дэви-Стюартсона также было изучено [25]. Напомним, что эволюционное уравнение является $P T$-симметричным, если оно инвариантно относительно совместного действия оператора четности $P(x \rightarrow-x)$ и оператора обращения времени (комплексного сопряжения) T. До сих пор ОЗР с ненулевыми граничными условиями рассматривалась только для $P T$-симметричного случая: $r(x, t)=\sigma q^{*}(-x, t)[26]$, т. е. для $P T$-симметричного НУШ

$$
i q_{t}=q_{x x}-2 \sigma q^{2}(x, t) q^{*}(-x, t), \quad \sigma=\mp 1 .
$$

Кроме РТ-симметричного НУШ (1.4), соответствующего симметрийной редукции $r(x, t)=\sigma q(-x,-t)$, была установлена интегрируемость следующего нелокального НУШ с обращением пространства-времени:

$$
i q_{t}(x, t)=q_{x x}(x, t)-2 \sigma q^{2}(x, t) q(-x,-t),
$$

а также изучена ОЗР для быстроубывающих граничных условий [22]. Замечательно то, что это уравнение является такой простой модификацией НУШ (1.3). Укажем два аспекта, касающиеся приведенного выше НУШ с обращением пространства-времени (1.5). Во-первых, коэффициент $\sigma$ может принимать значения не только \pm 1 , этот параметр может быть расположен где-нибудь на единичной окружности, т. е. 
$\sigma=e^{i \theta}, \theta \in \mathbb{R}$. Во-вторых, в этом случае, в отличие от НУШ или РТ-симметричного НУШ, коэффициент $\sigma$ можно убрать масштабированием. Именно преобразование уравнения (1.5) с помощью подстановки $q \rightarrow(-1 / \sigma)^{1 / 2} q$ позволяет без потери общности рассмотреть только случай $\sigma=1$.

Хотя МОЗР в присутствии симметрийной редукции $r(x, t)=\sigma q(-x,-t)$ был исследован для убывающих данных, ОЗР для нелокального НУШ с обращением пространства-времени с ненулевыми граничными условиями является пока новой и нерешенной задачей. Есть существенное различие между этим случаем и $P T$-симметричным случаем, рассмотренным в работе [26]. Эти нелокальные системы проявляют много интересных различий с их локальными аналогами.

В процессе построения решений с помощью МОЗР используются прямое и обратное рассеяния. Кратко этот процесс можно описать следующим образом. Исходя из нужным образом подобранного начального условия для потенциала $(q(x, t=0))$ начальные данные преобразуются с помощью прямой задачи рассеяния $X$ в данные рассеяния $S(k, t=0)$. Соответствующий оператор $T$ определяет эволюцию во времени данных рассеяния в любой момент времени $t \neq 0: S(k, t)$. Затем с помощью МОЗР данные рассеяния в любой момент времени $t$ используются для построения собственных функций линейных интегральных уравнений; по этой информации восстанавливается решение $q(x, t)$ нелинейного эволюционного уравнения.

В то время как МОЗР в качестве метода решения (линеаризации) начальной задачи Коши с убывающими, а иногда с ненулевыми граничными условиями на бесконечности использовался для многочисленных нелинейных уравнений, МОЗР с ненулевыми граничными условиями сложнее из-за более сложной структуры ветвления соответствующих линейных собственных функций.

В настоящей работе исследуются прямое и обратное спектральные преобразования, связанные с нелокальным НУШ с обращением пространства-времени со следующими ненулевыми граничными условиями:

$$
q(x, t) \rightarrow q_{0} e^{i\left(2 \sigma q_{0}^{2} t+\theta_{ \pm}\right)}, \quad x \rightarrow \pm \infty,
$$

где $\theta_{+}+\theta_{-}=0$ и $\theta_{+}+\theta_{-}=\pi$. Заметим, что некоторые свойства ненулевых граничных условий, связанных с НУШ с обращением пространства-времени (1.5), можно получить непосредственно. Если предположить, что $q \rightarrow q_{ \pm}(t)$ при $x \rightarrow \pm \infty$, то из уравнения (1.5) получим

$$
i q_{ \pm, t}(t)=-2 \sigma q_{ \pm}^{2}(t) q_{\mp}(-t) .
$$

Это означает, что

$$
q_{+}(t) q_{-}(-t)=C_{0}=\text { const },
$$

следовательно, уравнение (1.7) упрощается до

$$
i q_{ \pm, t}(t)=-2 \sigma C_{0} q_{ \pm}(t) .
$$

У этого уравнения есть решение

$$
q_{ \pm}(t)=\left|q_{ \pm}(0)\right| e^{2 i \sigma C_{0} t} e^{i \theta_{ \pm}},
$$

где $\theta_{ \pm}-$постоянные. Поскольку $C_{0}=\left|q_{+}(0)\right| \cdot\left|q_{-}(0)\right| \cdot e^{i\left(\theta_{+}+\theta_{-}\right)}, C_{0}$ является вещественной константой, если $\theta_{+}+\theta_{-}=0$ или $\theta_{+}+\theta_{-}=\pi$. В противном случае она 
комплексная и фон либо экспоненциально растет, либо убывает при $|t| \rightarrow \infty$. Без потери общности положим $q_{ \pm}(0)=q_{0}=$ const.

В разделе 3 при $\sigma=1, \theta_{+}+\theta_{-}=0$ получено следующее несингулярное односолитонное темное решение:

$$
q(x, t)=\frac{q_{0} e^{2 i q_{0}^{2} t}\left[e^{i \theta_{+}} \cdot e^{2 q_{0} x \sin \theta_{+}}+e^{-i \theta_{+}} \cdot e^{2 q_{0}^{2} t \sin \left(2 \theta_{+}\right)}\right]}{e^{2 q_{0} x \sin \theta_{+}}+e^{2 q_{0}^{2} t \sin \left(2 \theta_{+}\right)}} .
$$

В разделе 4 при $\sigma=1, \theta_{+}+\theta_{-}=\pi$ показано отсутствие соответствующего экспоненциально убывающего односолитонного решения, поскольку единственное собственное значение находится в непрерывном спектре. Простейший убывающий полностью безотражательный потенциал порождает двухсолитонное решение. Существуют несингулярные двухсолитонные решения.

В разделе 5 показано существование решений для нелокального НУШ с обращением пространства-времени, которые удовлетворяют следующим пространственно-зависимым граничным условиям:

$$
q(x, t) \rightarrow q_{0} e^{i\left(\alpha t+\beta x+\theta_{ \pm}\right)} \quad \text { при } \quad x \rightarrow \pm \infty,
$$

где как $\alpha$, так и $\beta$ вещественны. Для классического НУШ справедлива галилеева инвариантность.

Мы также можем найти новые типы решений для указанных выше уравнений, которые сингулярны вдоль пространственно-временных линий (см. [27]).

Наконец, подчеркнем, что МОЗР с ненулевыми граничными условиями, связанный с новым нелокальным НУШ с обращением пространства-времени, приводит к новым решениям. Рассмотренные здесь граничные задачи расширяют технику и границы применения МОЗР.

\section{2. НУШ С ОБРАЩЕНИЕМ ПРОСТРАНСТВА-ВРЕМЕНИ: СОВМЕСТНАЯ ЛИНЕЙНАЯ СИСТЕМА}

Нелокальное НУШ с обращением пространства-времени (1.5) связано со следующей совместной $(2 \times 2)$-системой:

$$
\begin{aligned}
& v_{x}=X v=\left(\begin{array}{cc}
-i k & q(x, t) \\
\sigma q(-x,-t) & i k
\end{array}\right) v \\
& v_{t}=T v=\left(\begin{array}{cc}
2 i k^{2}+i \sigma q(x, t) q(-x,-t) & -2 k q(x, t)-i q_{x}(x, t) \\
-2 \sigma k q(-x,-t)-\sigma i q_{x}(-x,-t) & -2 i k^{2}-i \sigma q(x, t) q(-x,-t)
\end{array}\right) v .
\end{aligned}
$$

Такие соотношения совместности хорошо известны (ср. с [8], [9]).

Мы видим, что при $x \rightarrow \pm \infty$ собственные функции пространственной задачи рассеяния асимптотически удовлетворяют соотношению

$$
\left(\begin{array}{l}
v_{1} \\
v_{2}
\end{array}\right)_{x}=\left(\begin{array}{cc}
-i k & q_{0} e^{i\left(\alpha t+\theta_{ \pm}\right)} \\
\sigma q_{0} e^{i\left(-\alpha t+\theta_{\mp}\right)} & i k
\end{array}\right)\left(\begin{array}{l}
v_{1} \\
v_{2}
\end{array}\right),
$$

где $\alpha=2 \sigma q_{0}^{2}$ (см. уравнение (1.6)). 


\section{3. НЕЛОКАЛЬНОЕ УРАВНЕНИЕ С ОБРАЩЕНИЕМ ПРОСТРАНСТВА-ВРЕМЕНИ: $\sigma=1$ И $\theta_{+}+\theta_{-}=0$}

3.1. Прямое рассеяние. Рассмотрим ненулевые граничные условия вида (1.6) при $\sigma=1$ и $\theta_{+}+\theta_{-}=0$. При таких условиях уравнение $(2.3)$ удобно свести к виду

$$
\frac{\partial^{2} v_{j}}{\partial x^{2}}=-\left(k^{2}-q_{0}^{2}\right) v_{j}, \quad j=1,2 .
$$

Каждое из двух уравнений имеет два линейно независимых решения $e^{i \lambda x}$ и $e^{-i \lambda x}$ при $|x| \rightarrow \infty$, где $\lambda=\sqrt{k^{2}-q_{0}^{2}}$. При этом переменная $k$ считается принадлежащей поверхности Римана $\mathbb{K}$, состоящей из двух листов $\mathbb{C}_{1}$ и $\mathbb{C}_{2}$ с разрезом комплексной плоскости вдоль $\left(-\infty,-q_{0}\right] \cup\left[q_{0},+\infty\right)$, а ее края склеены таким образом, что функция $\lambda(k)$ непрерывна в разрезе. Введем локальные полярные координаты

$$
k-q_{0}=r_{1} e^{i \theta_{1}}, \quad 0 \leqslant \theta_{1}<2 \pi, \quad k+q_{0}=r_{2} e^{i \theta_{2}}, \quad-\pi \leqslant \theta_{2}<\pi,
$$

где $r_{1}=\left|k-q_{0}\right|$ и $r_{2}=\left|k+q_{0}\right|$. Тогда функция $\lambda(k)$ становится однозначной на поверхности $\mathbb{K}$, т. е.

$$
\lambda(k)= \begin{cases}\lambda_{1}(k)=\left(r_{1} r_{2}\right)^{1 / 2} e^{i\left(\theta_{1}+\theta_{2}\right) / 2}, & k \in \mathbb{C}_{1}, \\ \lambda_{2}(k)=-\left(r_{1} r_{2}\right)^{1 / 2} e^{i\left(\theta_{1}+\theta_{2}\right) / 2}, & k \in \mathbb{C}_{2} .\end{cases}
$$

Более того, если $k \in \mathbb{C}_{1}$, то $\operatorname{Im} \lambda \geqslant 0 ;$ а если $k \in \mathbb{C}_{2}$, то $\operatorname{Im} \lambda \leqslant 0$. Таким образом, переменная $\lambda$ считается принадлежащей комплексной плоскости, состоящей из верхней полуплоскости $U_{+}: \operatorname{Im} \lambda \geqslant 0$ и нижней полуплоскости $U_{-}: \operatorname{Im} \lambda \leqslant 0$, склеенных вместе вдоль вещественной оси; переход происходит при $\operatorname{Im} \lambda=0$. Преобразование $k \rightarrow \lambda$ отображает $\mathbb{C}_{1}$ в $U_{+}, \mathbb{C}_{2}$ в $U_{-}$, разрез $\left(-\infty,-q_{0}\right] \cup\left[q_{0},+\infty\right)$ в вещественную ось, а точки $\pm q_{0}-$ в нуль (см. [26], стр. 6 , рис. 1,2$)$.

3.2. Собственные функции. Естественно ввести собственные функции, заданные с помощью следующих граничных условий:

$$
\phi(x, k) \sim w e^{-i \lambda x}, \quad \bar{\phi}(x, k) \sim \bar{w} e^{i \lambda x}
$$

при $x \rightarrow-\infty$,

$$
\psi(x, k) \sim v e^{i \lambda x}, \quad \bar{\psi}(x, k) \sim \bar{v} e^{-i \lambda x}
$$

при $x \rightarrow+\infty$. Подставив эти выражения в $(2.3)$, получим

$$
w=\left(\begin{array}{c}
\lambda+k \\
i q_{+}
\end{array}\right), \quad \bar{w}=\left(\begin{array}{c}
-i q_{-} \\
\lambda+k
\end{array}\right), \quad v=\left(\begin{array}{c}
-i q_{+} \\
\lambda+k
\end{array}\right), \quad \bar{v}=\left(\begin{array}{c}
\lambda+k \\
i q_{-}
\end{array}\right)
$$

что удовлетворяет граничным условиям. Эти граничные условия сводятся к хорошо известным граничным условиям в убывающем случае. Заметим, что функции $q_{+}, q_{-}$ в действительности зависят от времени; для функций $w, \bar{w}$, граничные условия для которых вычисляются на $-\infty$, имеем $q_{+}=q_{+}(-t), q_{-}=q_{-}(t)$, а для $v, \bar{v}$, граничные условия для которых вычисляются на $+\infty$, имеем $q_{+}=q_{+}(t), q_{-}=q_{-}(-t)$. Но для целей данного подраздела точная зависимость от времени не важна, поэтому мы используем упрощенные обозначения, опустив явную зависимость от времени. 
В дальнейшем анализе удобно рассматривать функции с постоянными граничными условиями. Определим ограниченные собственные функции следующим образом:

$$
\begin{aligned}
M(x, k) & =e^{i \lambda x} \phi(x, k), & & \bar{M}(x, k)=e^{-i \lambda x} \bar{\phi}(x, k), \\
N(x, k) & =e^{-i \lambda x} \psi(x, k), & \bar{N}(x, k) & =e^{i \lambda x} \bar{\psi}(x, k) .
\end{aligned}
$$

Собственные функции можно представить с помощью следующих интегральных уравнений:

$$
\begin{aligned}
& M(x, k)=\left(\begin{array}{c}
\lambda+k \\
i q_{+}
\end{array}\right)+\int_{-\infty}^{+\infty} G_{-}\left(x-x^{\prime}, k\right)\left(\left(Q-Q_{-}\right) M\right)\left(x^{\prime}, k\right) d x^{\prime}, \\
& \bar{M}(x, k)=\left(\begin{array}{c}
-i q_{-} \\
\lambda+k
\end{array}\right)+\int_{-\infty}^{+\infty} \bar{G}_{-}\left(x-x^{\prime}, k\right)\left(\left(Q-Q_{-}\right) M\right)\left(x^{\prime}, k\right) d x^{\prime}, \\
& N(x, k)=\left(\begin{array}{c}
-i q_{+} \\
\lambda+k
\end{array}\right)+\int_{-\infty}^{+\infty} G_{+}\left(x-x^{\prime}, k\right)\left(\left(Q-Q_{+}\right) M\right)\left(x^{\prime}, k\right) d x^{\prime}, \\
& \bar{N}(x, k)=\left(\begin{array}{c}
\lambda+k \\
i q_{-}
\end{array}\right)+\int_{-\infty}^{+\infty} \bar{G}_{+}\left(x-x^{\prime}, k\right)\left(\left(Q-Q_{+}\right) M\right)\left(x^{\prime}, k\right) d x^{\prime} .
\end{aligned}
$$

Используя метод преобразования Фурье, получим

$$
\begin{aligned}
& G_{-}(x, k)=\frac{\theta(x)}{2 \lambda}\left[\left(1+e^{2 i \lambda x}\right) \lambda I-i\left(e^{2 i \lambda x}-1\right)\left(i k J+Q_{-}\right)\right], \\
& \bar{G}_{-}(x, k)=\frac{\theta(x)}{2 \lambda}\left[\left(1+e^{-2 i \lambda x}\right) \lambda I+i\left(e^{-2 i \lambda x}-1\right)\left(i k J+Q_{-}\right)\right], \\
& G_{+}(x, k)=-\frac{\theta(-x)}{2 \lambda}\left[\left(1+e^{-2 i \lambda x}\right) \lambda I+i\left(e^{-2 i \lambda x}-1\right)\left(i k J+Q_{+}\right)\right], \\
& \bar{G}_{+}(x, k)=-\frac{\theta(-x)}{2 \lambda}\left[\left(1+e^{2 i \lambda x}\right) \lambda I-i\left(e^{2 i \lambda x}-1\right)\left(i k J+Q_{+}\right)\right],
\end{aligned}
$$

где $\theta(x)$ - функция Хевисайда, т. е. $\theta(x)=1$ при $x>0$ и $\theta(x)=0$ при $x<0$.

ОПРЕДЕЛЕНИЕ 1. Будем говорить, что $f \in L^{1}(\mathbb{R})$, если $\int_{-\infty}^{+\infty}|f(x)| d x<\infty$, и $f \in L^{1, N}(\mathbb{R})$, если $\int_{-\infty}^{+\infty}|f(x)| \cdot(1+|x|)^{N} d x<\infty$, где $N=1,2, \ldots$ - заданное положительное целое число.

Тогда получим следующий результат.

Теорема 1. Предположим, что элементы $Q-Q_{ \pm}$принадлежат $L^{1}(\mathbb{R})$. Тогда для каждого $x \in \mathbb{R}$ собственные функции $M(x, k)$ u $N(x, k)$ непрерывны при $k \in$ $\overline{\mathbb{C}}_{1} \backslash\left\{ \pm q_{0}\right\}$ и аналитичнъ при $k \in \mathbb{C}_{1}$, а функиии $\bar{M}(x, k)$ и $\bar{N}(x, k)$ непреръвнъ при $k \in \overline{\mathbb{C}}_{2} \backslash\left\{ \pm q_{0}\right\}$ и аналитичнъ при $k \in \mathbb{C}_{2}$. Кроме того, если элементы $Q-$ $Q_{ \pm}$принадлежат $L^{1,2}(\mathbb{R})$, тогда для любого $x \in \mathbb{R}$ собственные функции $M(x, k)$ u $N(x, k)$ непрерывны при $k \in \overline{\mathbb{C}}_{1}$ и аналитичнъ при $k \in \mathbb{C}_{1}$, а функции $\bar{M}(x, k)$ $u \bar{N}(x, k)$ непрерывнъ при $k \in \overline{\mathbb{C}}_{2}$ и аналитичнъ при $k \in \mathbb{C}_{2}$. Здесъ $\overline{\mathbb{C}}_{j}, j=1,2$, являются замыканиями листов $\mathbb{C}_{j}, j=1,2$.

Доказательство основано на рядах Неймана с использованием условий $\operatorname{Im} \lambda \geqslant 0$ или $\operatorname{Im} \lambda>0$; оно аналогично доказательству, приведенному в работе [26]. 
ЗАмЕчАниЕ 1. Как это было сделано в теореме 3.2 в работе [26], можно переписать функции Грина в терминах проекторов, например

$$
M(x, k)=\left(\begin{array}{c}
\lambda+k \\
-i q_{+}^{*}
\end{array}\right)+\int_{-\infty}^{x}\left[P_{-i \lambda}^{-}+e^{2 i \lambda\left(x-x^{\prime}\right)} P_{i \lambda}^{-}\right]\left(\left(Q-Q_{-}\right) M\right)\left(x^{\prime}, k\right) d x^{\prime},
$$

где

$$
P_{-i \lambda}^{ \pm}(k)=\frac{1}{2 \lambda}\left(\begin{array}{cc}
\lambda+k & i q_{ \pm} \\
i q_{\mp} & \lambda-k
\end{array}\right), \quad P_{i \lambda}^{ \pm}(k)=\frac{1}{2 \lambda}\left(\begin{array}{cc}
\lambda-k & -i q_{ \pm} \\
-i q_{\mp} & \lambda+k
\end{array}\right) .
$$

Чтобы распространить свойство непрерывности в точку $k= \pm q_{0}$, перепишем выражение $P_{-i \lambda}^{-}+e^{2 i \lambda\left(x-x^{\prime}\right)} P_{i \lambda}^{-}$в виде $I_{2}+\left[e^{2 i \lambda\left(x-x^{\prime}\right)}-1\right] P_{i \lambda}^{-}$и используем оценку

$$
\begin{aligned}
\left\|I_{2}+\left[e^{2 i \lambda\left(x-x^{\prime}\right)}-1\right] P_{i \lambda}^{-}\right\| \leqslant 1+2\left|x-x^{\prime}\right| \cdot|k| & \leqslant \max \{1,2|k|\}(1+|x|)\left(1+\left|x^{\prime}\right|\right) \leqslant \\
& \leqslant \max \{1,2|k|\}(1+|x|)^{2},
\end{aligned}
$$

поэтому нужны элементы $Q-Q_{ \pm}$, принадлежащие $L^{1,2}(\mathbb{R})$.

3.3. Данные рассеяния. Две собственные функции $\phi, \bar{\phi}, \psi, \bar{\psi}$ являются линейно независимыми. Действительно, если $u(x, k)=\left(u_{1}(x, k), u_{2}(x, k)\right)^{\mathrm{T}}$ и $v(x, k)=$ $\left(v_{1}(x, k), v_{2}(x, k)\right)^{\mathrm{T}}$ - любые два решения уравнения $(2.1)$ при $\sigma=1$, имеем

$$
\frac{d}{d x} W(u, v)=0
$$

где вронскиан $W(u, v)$ решений $u$ и $v$ имеет вид $W(u, v)=u_{1} v_{2}-u_{2} v_{1}$. Из асимптотик (3.4) и (3.5) следует, что

$$
\begin{aligned}
W(\phi, \bar{\phi}) & =\lim _{x \rightarrow-\infty} W(\phi(x, k), \bar{\phi}(x, k))=2 \lambda(\lambda+k), \\
W(\psi, \bar{\psi}) & =\lim _{x \rightarrow+\infty} W(\psi(x, k), \bar{\psi}(x, k))=-2 \lambda(\lambda+k),
\end{aligned}
$$

это доказывает, что функции $\phi(x, k)$ и $\bar{\phi}(x, k)$ являются линейно независимыми, так же как и $\psi$ и $\bar{\psi}$, с единственным исключением точек ветвления $\pm q_{0}$. Следовательно, можно представить $\phi(x, k)$ и $\bar{\phi}(x, k)$ в виде линейных комбинаций $\psi(x, k)$ и $\bar{\psi}(x, k)$, или наоборот. Таким образом, соотношения

$$
\begin{aligned}
& \phi(x, k)=b(k) \psi(x, k)+a(k) \bar{\psi}(x, k), \\
& \bar{\phi}(x, k)=\bar{a}(k) \psi(x, k)+\bar{b}(k) \bar{\psi}(x, k)
\end{aligned}
$$

справедливы для любого $k$, при котором существуют все четыре собственные функции. Комбинируя (3.14) и (3.15), можно получить, что данные рассеяния удовлетворяют следующему характеристическому уравнению:

$$
a(k) \bar{a}(k)-b(k) \bar{b}(k)=1 .
$$


Данные рассеяния можно выразить через вронскианы собственных функций, т. е.

$$
\begin{aligned}
& a(k)=\frac{W(\phi(x, k), \psi(x, k))}{W(\bar{\psi}(x, k), \psi(x, k))}=\frac{W(\phi(x, k), \psi(x, k))}{2 \lambda(\lambda+k)}, \\
& \bar{a}(k)=-\frac{W(\bar{\phi}(x, k), \bar{\psi}(x, k))}{W(\bar{\psi}(x, k), \psi(x, k))}=-\frac{W(\bar{\phi}(x, k), \bar{\psi}(x, k))}{2 \lambda(\lambda+k)}, \\
& b(k)=-\frac{W(\phi(x, k), \bar{\psi}(x, k))}{W(\bar{\psi}(x, k), \psi(x, k))}=-\frac{W(\phi(x, k), \bar{\psi}(x, k))}{2 \lambda(\lambda+k)}, \\
& \bar{b}(k)=\frac{W(\bar{\phi}(x, k), \psi(x, k))}{W(\bar{\psi}(x, k), \psi(x, k))}=\frac{W(\bar{\phi}(x, k), \psi(x, k))}{2 \lambda(\lambda+k)} .
\end{aligned}
$$

Тогда ввиду аналитичности собственных функций получим следующую теорему.

Теорема 2. Предположим, что элементы $Q-Q_{ \pm}$принадлежат $L^{1}(\mathbb{R})$, тогда функция $а(k)$ непрерьвна при $k \in \overline{\mathbb{C}}_{1} \backslash\left\{ \pm q_{0}\right\}$ и аналитична при $k \in \mathbb{C}_{1}$, а функиия $\bar{a}(k)$ непрерьвна при $k \in \overline{\mathbb{C}}_{2} \backslash\left\{ \pm q_{0}\right\}$ и аналитична при $k \in \mathbb{C}_{2}$. Более того, бункции $b(k)$ и $\bar{b}(k)$ непрерьвны в $k \in\left(-\infty,-q_{0}\right) \cup\left(q_{0},+\infty\right)$. Кроме того, если элементы $Q-Q_{ \pm}$принадлежат $L^{1,2}(\mathbb{R})$, то функция $a(k) \lambda(k)$ непрерывна при $k \in \overline{\mathbb{C}}_{1}$ и аналитична при $k \in \mathbb{C}_{1}$, а $\bar{a}(k) \lambda(k)$ непрерьвна при $k \in \overline{\mathbb{C}}_{2}$ и аналитична при $k \in \mathbb{C}_{2}$. Более того, $b(k) \lambda(k)$ и $\bar{b}(k) \lambda(k)$ непрерывны при $k \in \mathbb{R}$. Если элементы $Q-Q_{ \pm}$растут не бъстрее, чем $e^{-a x^{2}}$, где а - положительное вещественное число, то $а(k) \lambda(k), \bar{a}(k) \lambda(k), b(k) \lambda(k)$ и $\bar{b}(k) \lambda(k)$ аналитичны при $k \in \mathbb{K}$.

Доказательство теоремы 2 следует из вронскианных соотношений (см. также [26]).

Заметим, что соотношения (3.16) и (3.17) можно представить в виде

$$
\mu(x, k)=\rho(k) e^{2 i \lambda x} N(x, k)+\bar{N}(x, k), \quad \bar{\mu}(x, k)=N(x, k)+\bar{\rho}(k) e^{-2 i \lambda x} \bar{N}(x, k),
$$

где $\mu(x, k)=M(x, k) a^{-1}(k), \bar{\mu}(x, k)=\bar{M}(x, k) \bar{a}^{-1}(k), \rho(k)=b(k) a^{-1}(k)$ и $\bar{\rho}(k)=$ $\bar{b}(k) \bar{a}^{-1}(k)$. Введем $(2 \times 2)$-матрицы

$$
m_{+}(x, k)=(\mu(x, k), N(x, k)), \quad m_{-}(x, k)=(\bar{N}(x, k), \bar{\mu}(x, k)),
$$

которые мероморфны в $\mathbb{C}_{1}$ и $\mathbb{C}_{2}$ соответственно. Следовательно, можно представить задачу Римана-Гильберта или условие на "скачок" на плоскости $k$ в виде уравнения

$$
m_{+}(x, k)-m_{-}(x, k)=m_{-}(x, k)\left(\begin{array}{cc}
-\rho(k) \bar{\rho}(k) & -\bar{\rho}(k) e^{-2 i \lambda x} \\
\rho(k) e^{2 i \lambda x} & 0
\end{array}\right)
$$

на контуре $\Sigma: k \in\left(-\infty,-q_{0}\right] \cup\left[q_{0},+\infty\right)$. Напомним, что на листе $\mathbb{C}_{1}$ справедливо $\operatorname{Im} \lambda>0$, на листе $\mathbb{C}_{2}-\operatorname{Im} \lambda<0$, а на контуре $\Sigma-\operatorname{Im} \lambda=0$.

3.4. Симметрийные редукции. Симметрия потенциала порождает симметрию между собственными функциями. Действительно, если функция $v(x, k)=$ $\left(v_{1}(x, k), v_{2}(x, k)\right)^{\mathrm{T}}-$ решение уравнения $(2.1)$ при $\sigma=1$, то $\left(v_{2}(-x, k),-v_{1}(-x, k)\right)^{\mathrm{T}}$ также является решением уравнения (2.1) при $\sigma=1$. Принимая во внимание граничные условия (3.6), получим

$$
\psi(x, k)=\left(\begin{array}{cc}
0 & -1 \\
1 & 0
\end{array}\right) \phi(-x, k), \quad \bar{\psi}(x, k)=\left(\begin{array}{cc}
0 & 1 \\
-1 & 0
\end{array}\right) \bar{\phi}(-x, k) .
$$


Используя формулы (3.7), можно получить симметрийные связи для собственных функций:

$$
N(x, k)=\left(\begin{array}{rr}
0 & -1 \\
1 & 0
\end{array}\right) M(-x, k), \quad \bar{N}(x, k)=\left(\begin{array}{rr}
0 & 1 \\
-1 & 0
\end{array}\right) \bar{M}(-x, k) .
$$

Из вронскианного представления данных рассеяния и указанной выше симметрийной редукции имеем

$$
\bar{b}(k)=b(k) .
$$

3.5. Униформизующие координаты. Прежде чем обсуждать нули данных рассеяния и решать обратную задачу, введем униформизующую переменную $z$, определенную конформным отображением:

$$
z=z(k)=k+\lambda(k),
$$

где $\lambda=\sqrt{k^{2}-q_{0}^{2}}$, а обратное отображение имеет вид $k=k(z)=\left(z+q_{0}^{2} / z\right) / 2$. Тогда $\lambda(z)=\left(z-q_{0}^{2} / z\right) / 2$. Заметим, что справедливы следующие утверждения:

$1)$ верхний лист $\mathbb{C}_{1}$ и нижний лист $\mathbb{C}_{2}$ римановой поверхности $\mathbb{K}$ отображаются соответственно в верхнюю $\mathbb{C}^{+}$и нижнюю $\mathbb{C}^{-}$полуплоскости комплексной плоскости $z$;

2) разрез $\left(-\infty,-q_{0}\right] \cup\left[q_{0},+\infty\right)$ на римановой поверхности отображается в вещественную ось $z$;

3 ) сегменты $\left[-q_{0}, q_{0}\right]$ на $\mathbb{C}_{1}$ и $\mathbb{C}_{2}$ отображаются соответственно на верхнюю и нижнюю полуокружности радиуса $q_{0}$ с центром в начале координат на комплексной плоскости $z$.

Из теоремы 1 следует, что собственные функции $M(x, z)$ и $N(x, z)$ аналитичны в верхней полуплоскости $z$, т. е. $z \in \mathbb{C}^{+}$, а функции $\bar{M}(x, z)$ и $\bar{N}(x, z)$ аналитичны в нижней полуплоскости $z$, т. е. $z \in \mathbb{C}^{-}$. Более того, согласно теореме 2 функция $a(z)$ аналитична в верхней полуплоскости $z: z \in \mathbb{C}^{+}$, а $\bar{a}(z)$ аналитична в нижней полуплоскости $z: z \in \mathbb{C}^{-}$.

3.6. Симметрии в униформизующих координатах. Из указанных выше симметрий собственных функций имеем

$$
\psi(x, z)=\left(\begin{array}{rr}
0 & -1 \\
1 & 0
\end{array}\right) \phi(-x, z), \quad \bar{\psi}(x, z)=\left(\begin{array}{rr}
0 & 1 \\
-1 & 0
\end{array}\right) \bar{\phi}(-x, z) .
$$

Далее, если $z \rightarrow q_{0}^{2} / z$, то $(k, \lambda) \rightarrow(k,-\lambda)$. Следовательно,

$$
\phi\left(x, \frac{q_{0}^{2}}{z}\right)=\frac{q_{0}^{2} / z}{-i q_{-}} \bar{\phi}(x, z), \quad \psi\left(x, \frac{q_{0}^{2}}{z}\right)=\frac{-i q_{+}}{z} \bar{\psi}(x, z), \quad \operatorname{Im} z<0 .
$$

Аналогично получим

$$
\begin{aligned}
& N(x, z)=\left(\begin{array}{cc}
0 & -1 \\
1 & 0
\end{array}\right) M(-x, z), \quad \bar{N}(x, z)=\left(\begin{array}{cc}
0 & 1 \\
-1 & 0
\end{array}\right) \bar{M}(-x, z), \\
& \bar{b}(z)=b(z) ; \quad a\left(\frac{q_{0}^{2}}{z}\right)=e^{2 i \theta+} \bar{a}(z), \quad \operatorname{Im} z<0 ; \quad b\left(\frac{q_{0}^{2}}{z}\right)=-\bar{b}(z) .
\end{aligned}
$$


3.7. Асимптотическое поведение собственных функций и данных рассеяния. Чтобы решить обратную задачу, нужно определить асимптотическое поведение собственных функций и данных рассеяния как при $z \rightarrow \infty$, так и при $z \rightarrow 0$. Из интегральных уравнений (в терминах функций Грина) имеем

$$
\begin{gathered}
\bar{N}(x, z) \sim\left(\begin{array}{c}
z \frac{q(x)}{q_{+}} \\
i q_{-}
\end{array}\right), \quad z \rightarrow 0, \\
a(z)=\left\{\begin{array}{ll}
1, & z \rightarrow \infty, \\
e^{2 i \theta_{+}}, & z \rightarrow 0,
\end{array} \quad \bar{a}(z)= \begin{cases}1, & z \rightarrow \infty, \\
e^{-2 i \theta_{+}}, & z \rightarrow 0,\end{cases} \right. \\
\lim _{z \rightarrow \infty} z b(z)=0, \quad \lim _{z \rightarrow 0} \frac{b(z)}{z^{2}}=0 .
\end{gathered}
$$

\section{8. Проблема Римана-Гильберта в униформизующих координатах.}

3.8.1. Левосторонняя задача рассеяния. Чтобы учесть поведение собственных функций, представим условия на “скачок" на вещественной оси $z$ в виде

$$
\begin{aligned}
& \frac{M(x, z)}{z a(z)}-\frac{\bar{N}(x, z)}{z}=\rho(z) e^{i\left(z-q_{0}^{2} / z\right) x} \frac{N(x, z)}{z}, \\
& \frac{\bar{M}(x, z)}{z \bar{a}(z)}-\frac{N(x, z)}{z}=\bar{\rho}(z) e^{-i\left(z-q_{0}^{2} / z\right) x} \frac{\bar{N}(x, z)}{z} .
\end{aligned}
$$

Таким образом, функции будут ограничены на бесконечности, хотя и имеют дополнительный полюс при $z=0$. Заметим, что $M(x, z) / a(z)$, как функция от $z$, определена в верхней полуплоскости $\mathbb{C}^{+}$, где у нее по предположению имеются простые полюсы $z_{j}$, т. е. $a\left(z_{j}\right)=0$, а $\bar{M}(x, z) / \bar{a}(z)$ определена в нижней полуплоскости $\mathbb{C}^{-}$, где у нее имеются простые полюсы $\bar{z}_{j}$, т.е. $\bar{a}\left(\bar{z}_{j}\right)=0$. Действительно, это аналогично случаю НУШ, где функции $a(z), \bar{a}(z)$ могут иметь кратные нули и/или нули на вещественной оси [8]. Понятие "собственного нуля" исключает эти частные возможности. В нулях $a, \bar{a}$ имеем

$$
M\left(x, z_{j}\right)=b\left(z_{j}\right) e^{i\left(z_{j}-q_{0}^{2} / z_{j}\right) x} N\left(x, z_{j}\right), \quad \bar{M}\left(x, \bar{z}_{j}\right)=\bar{b}\left(\bar{z}_{j}\right) e^{-i\left(\bar{z}_{j}-q_{0}^{2} / \bar{z}_{j}\right) x} \bar{N}\left(x, \bar{z}_{j}\right) .
$$

Тогда, вычитая значение на бесконечности, индуцированный полюс в начале координат и полюсы (предположительно простые) в верхней и нижней полуплоскостях соответственно при $a\left(z_{j}\right)=0, j=1,2, \ldots, J$, и $\bar{a}\left(\bar{z}_{j}\right), j=1,2, \ldots, \bar{J}$ (ниже мы увидим, что $J=\bar{J})$, получим

$$
\begin{aligned}
& {\left[\frac{M(x, z)}{z a(z)}-\left(\begin{array}{l}
1 \\
0
\end{array}\right)-\frac{1}{z}\left(\begin{array}{c}
0 \\
i q_{-}
\end{array}\right)-\sum_{j=1}^{J} \frac{M\left(x, z_{j}\right)}{\left(z-z_{j}\right) z_{j} a^{\prime}\left(z_{j}\right)}\right]-} \\
& -\left[\frac{\bar{N}(x, z)}{z}-\left(\begin{array}{c}
1 \\
0
\end{array}\right)-\frac{1}{z}\left(\begin{array}{c}
0 \\
i q_{-}
\end{array}\right)-\sum_{j=1}^{J} \frac{b\left(z_{j}\right) e^{i\left(z_{j}-q_{0}^{2} / z_{j}\right) x} N\left(x, z_{j}\right)}{\left(z-z_{j}\right) z_{j} a^{\prime}\left(z_{j}\right)}\right]= \\
& =\rho(z) e^{i\left(z-q_{0}^{2} / z\right) x} \frac{N(x, z)}{z},
\end{aligned}
$$




$$
\begin{aligned}
& {\left[\frac{\bar{M}(x, z)}{z \bar{a}(z)}-\left(\begin{array}{c}
0 \\
1
\end{array}\right)-\frac{1}{z}\left(\begin{array}{c}
-i q_{+} \\
0
\end{array}\right)-\sum_{j=1}^{\bar{J}} \frac{\bar{M}\left(x, \bar{z}_{j}\right)}{\left(z-\bar{z}_{j}\right) \bar{z}_{j} a^{\prime}\left(\bar{z}_{j}\right)}\right]-} \\
& \quad-\left[\frac{N(x, z)}{z}-\left(\begin{array}{c}
0 \\
1
\end{array}\right)-\frac{1}{z}\left(\begin{array}{c}
-i q_{+} \\
0
\end{array}\right)-\sum_{j=1}^{\bar{J}} \frac{\bar{b}\left(\bar{z}_{j}\right) e^{-i\left(\bar{z}_{j}-q_{0}^{2} / \bar{z}_{j}\right) x} \bar{N}\left(x, \bar{z}_{j}\right)}{\left(z-\bar{z}_{j}\right) \bar{z}_{j} \bar{a}^{\prime}\left(\bar{z}_{j}\right)}\right]= \\
& \quad=\bar{\rho}(z) e^{-i\left(z-q_{0}^{2} / z\right) x} \frac{\bar{N}(x, z)}{z} .
\end{aligned}
$$

Введем теперь проекторы

$$
P_{ \pm}(f)(z)=\frac{1}{2 \pi i} \int_{-\infty}^{+\infty} \frac{f(\xi)}{\xi-(z \pm i 0)} d \xi
$$

которые хорошо определены для любой функции $f(\xi)$, интегрируемой на вещественной оси. Если функции $f_{ \pm}(\xi)$ аналитичны соответственно в верхней и нижней полуплоскостях $z$ и убывают при больших $\xi$, то

$$
P_{ \pm}\left(f_{ \pm}\right)(z)= \pm f_{ \pm}(z), \quad P_{\mp}\left(f_{ \pm}\right)(z)=0 .
$$

Применяя оператор $P_{-}$к $(3.34)$, а $P_{+}$к $(3.35)$, получим

$$
\begin{aligned}
\bar{N}(x, z)= & \left(\begin{array}{c}
z \\
i q_{-}
\end{array}\right)+\sum_{j=1}^{J} \frac{z b\left(z_{j}\right) e^{i\left(z_{j}-q_{0}^{2} / z_{j}\right) x} N\left(x, z_{j}\right)}{\left(z-z_{j}\right) z_{j} a^{\prime}\left(z_{j}\right)}+ \\
& +\frac{z}{2 \pi i} \int_{-\infty}^{+\infty} \frac{\rho(\xi)}{\xi(\xi-z)} e^{i\left(\xi-q_{0}^{2} / \xi\right) x} N(x, \xi) d \xi, \\
N(x, z)= & \left(\begin{array}{c}
-i q_{+} \\
z
\end{array}\right)+\sum_{j=1}^{\bar{J}} \frac{z \bar{b}\left(\bar{z}_{j}\right) e^{-i\left(\bar{z}_{j}-q_{0}^{2} / \bar{z}_{j}\right) x} \bar{N}\left(x, \bar{z}_{j}\right)}{\left(z-\bar{z}_{j}\right) \bar{z}_{j} \bar{a}^{\prime}\left(\bar{z}_{j}\right)}- \\
& -\frac{z}{2 \pi i} \int_{-\infty}^{+\infty} \frac{\bar{\rho}(\xi)}{\xi(\xi-z)} e^{-i\left(\xi-q_{0}^{2} / \xi\right) x} \bar{N}(x, \xi) d \xi .
\end{aligned}
$$

Поскольку существуют симметрии между собственными функциями, определенными на обеих бесконечностях $\pm \infty$, приступим к выводу интегральных уравнений для обратного рассеяния, определенных на правом конце.

3.8.2. Правосторонняя задача рассеяния. Правостороннюю задачу рассеяния можно представить в виде

$$
\psi(x, z)=\alpha(z) \bar{\phi}(x, z)+\beta(z) \phi(x, z), \quad \bar{\psi}(x, z)=\bar{\alpha}(z) \phi(x, z)+\bar{\beta}(z) \bar{\phi}(x, z),
$$

где $\alpha(z), \bar{\alpha}(z), \beta(z)$ и $\bar{\beta}(z)$ являются правосторонними данными рассеяния. Более того, можно получить правосторонние и левосторонние данные рассеяния, удовлетворяющие следующим соотношениям:

$$
\bar{\alpha}(z)=\bar{a}(z), \quad \alpha(z)=a(z), \quad \bar{\beta}(z)=-b(z), \quad \beta(z)=-\bar{b}(z) .
$$


Таким образом, ввиду симметрийных связей между данными рассеяния имеем

$$
\begin{aligned}
& \frac{N(x, z)}{z a(z)}-\frac{\bar{M}(x, z)}{z}=\rho^{*}\left(-z^{*}\right) e^{-i\left(z-q_{0}^{2} / z\right) x} \frac{M(x, z)}{z}, \\
& \frac{\bar{N}(x, z)}{z \bar{a}(z)}-\frac{M(x, z)}{z}=\bar{\rho}^{*}\left(-z^{*}\right) e^{i\left(z-q_{0}^{2} / z\right) x} \frac{\bar{M}(x, z)}{z}
\end{aligned}
$$

так что функции ограничены на бесконечности, имея при этом дополнительный полюс при $z=0$. Заметим, что $N(x, z) / a(z)$, как функция от $z$, определена в верхней полуплоскости $\mathbb{C}^{+}$, где (по предположению) она имеет простые полюсы $z_{j}$, т. е. $a\left(z_{j}\right)=0$, а функция $\bar{N}(x, z) / \bar{a}(z)$ определена в нижней полуплоскости $\mathbb{C}^{-}$, где она имеет простые полюсы $\bar{z}_{j}$, т. е. $\bar{a}\left(\bar{z}_{j}\right)=0$. В нулях функций $a, \bar{a}$ имеем

$$
\begin{aligned}
& N\left(x, z_{j}\right)=-\bar{b}\left(z_{j}\right) M\left(x, z_{j}\right) e^{-i\left(z_{j}-q_{0}^{2} / z_{j}\right) x} \\
& \bar{N}\left(x, \bar{z}_{j}\right)=-b\left(\bar{z}_{j}\right) \bar{M}\left(x, \bar{z}_{j}\right) e^{i\left(\bar{z}_{j}-q_{0}^{2} / \bar{z}_{j}\right) x}
\end{aligned}
$$

Тогда, как и ранее, вычитая значение на бесконечности, индуцированный полюс в начале координат и полюсы (предположительно простые) в верхней и нижней полуплоскостях соответственно при $a\left(z_{j}\right)=0, j=1,2, \ldots, J$, и $\bar{a}\left(\bar{z}_{j}\right), j=1,2, \ldots, \bar{J}$, получим

$$
\begin{aligned}
& {\left[\frac{N(x, z)}{z a(z)}-\left(\begin{array}{l}
0 \\
1
\end{array}\right)-\frac{1}{z}\left(\begin{array}{c}
-i q_{-} \\
0
\end{array}\right)-\sum_{j=1}^{J} \frac{N\left(x, z_{j}\right)}{\left(z-z_{j}\right) z_{j} a^{\prime}\left(z_{j}\right)}\right]-} \\
& -\left[\frac{\bar{M}(x, z)}{z}-\left(\begin{array}{l}
0 \\
1
\end{array}\right)-\frac{1}{z}\left(\begin{array}{c}
-i q_{-} \\
0
\end{array}\right)-\sum_{j=1}^{J} \frac{-\bar{b}\left(z_{j}\right) M\left(x, z_{j}\right) e^{-i\left(z_{j}-q_{0}^{2} / z_{j}\right) x}}{\left(z-z_{j}\right) z_{j} a^{\prime}\left(z_{j}\right)}\right]= \\
& =\rho^{*}\left(-z^{*}\right) e^{-i\left(z-q_{0}^{2} / z\right) x} \frac{M(x, z)}{z}, \\
& {\left[\frac{\bar{N}(x, z)}{z \bar{a}(z)}-\left(\begin{array}{l}
1 \\
0
\end{array}\right)-\frac{1}{z}\left(\begin{array}{c}
0 \\
i q_{+}
\end{array}\right)-\sum_{j=1}^{\bar{J}} \frac{\bar{N}\left(x, \bar{z}_{j}\right)}{\left(z-\bar{z}_{j}\right) \bar{z}_{j} \bar{a}^{\prime}\left(\bar{z}_{j}\right)}\right]-} \\
& -\left[\frac{M(x, z)}{z}-\left(\begin{array}{l}
1 \\
0
\end{array}\right)-\frac{1}{z}\left(\begin{array}{c}
0 \\
i q_{+}
\end{array}\right)-\sum_{j=1}^{\bar{J}} \frac{-b\left(\bar{z}_{j}\right) \bar{M}\left(x, \bar{z}_{j}\right) e^{i\left(\bar{z}_{j}-q_{0}^{2} / \bar{z}_{j}\right) x}}{\left(z-\bar{z}_{j}\right) \bar{z}_{j} \bar{a}^{\prime}\left(\bar{z}_{j}\right)}\right]= \\
& =\bar{\rho}^{*}\left(-z^{*}\right) e^{i\left(z-q_{0}^{2} / z\right) x} \frac{\bar{M}(x, z)}{z} .
\end{aligned}
$$

Применяя операторы $P_{-}$к $(3.44)$ и $P_{+}$к $(3.45)$, получим

$$
\begin{aligned}
\bar{M}(x, z)= & \left(\begin{array}{c}
-i q_{-} \\
z
\end{array}\right)+\sum_{j=1}^{J} \frac{-z \bar{b}\left(z_{j}\right) M\left(x, z_{j}\right) e^{-i\left(z_{j}-q_{0}^{2} / z_{j}\right) x}}{\left(z-z_{j}\right) z_{j} a^{\prime}\left(z_{j}\right)}+ \\
& +\frac{z}{2 \pi i} \int_{-\infty}^{+\infty} \frac{\rho^{*}(-\xi)}{\xi(\xi-z)} e^{-i\left(\xi-q_{0}^{2} / \xi\right) x} M(x, \xi) d \xi \\
M(x, z)= & \left(\begin{array}{c}
z \\
i q_{+}
\end{array}\right)+\sum_{j=1}^{\bar{J}} \frac{-z b\left(\bar{z}_{j}\right) \bar{M}\left(x, \bar{z}_{j}\right) e^{i\left(\bar{z}_{j}-q_{0}^{2} / \bar{z}_{j}\right) x}}{\left(z-\bar{z}_{j}\right) \bar{z}_{j} \bar{a}^{\prime}\left(\bar{z}_{j}\right)}- \\
& -\frac{z}{2 \pi i} \int_{-\infty}^{+\infty} \frac{\bar{\rho}^{*}(-\xi)}{\xi(\xi-z)} e^{i\left(\xi-q_{0}^{2} / \xi\right) x} \bar{M}(x, \xi) d \xi
\end{aligned}
$$


3.9. Восстановление потенциалов. Для восстановления потенциала используем асимптотики. Например, из уравнения (3.30) получаем $\bar{N}_{1}(x, z) / z \sim q(x) / q_{+}$ при $z \rightarrow 0$. В силу (3.38) имеем

$$
\begin{aligned}
\frac{\bar{N}_{1}(x, z)}{z} \sim 1 & +\sum_{j=1}^{J} \frac{b\left(z_{j}\right) e^{i\left(z_{j}-q_{0}^{2} / z_{j}\right) x}}{-z_{j}^{2} a^{\prime}\left(z_{j}\right)} N_{1}\left(x, z_{j}\right)+ \\
& +\frac{1}{2 \pi i} \int_{-\infty}^{+\infty} \frac{\rho(\xi)}{\xi^{2}} e^{i\left(\xi-q_{0}^{2} / \xi\right) x} N_{1}(x, \xi) d \xi
\end{aligned}
$$

при $z \rightarrow 0$. Следовательно,

$$
\begin{aligned}
q(x)=q_{+}[1 & +\sum_{j=1}^{J} \frac{b\left(z_{j}\right) e^{i\left(z_{j}-q_{0}^{2} / z_{j}\right) x}}{-z_{j}^{2} a^{\prime}\left(z_{j}\right)} N_{1}\left(x, z_{j}\right)+ \\
& \left.+\frac{1}{2 \pi i} \int_{-\infty}^{+\infty} \frac{\rho(\xi)}{\xi^{2}} e^{i\left(\xi-q_{0}^{2} / \xi\right) x} N_{1}(x, \xi) d \xi\right]
\end{aligned}
$$

3.10. Замыкание системы. Из $a\left(q_{0}^{2} / z\right)=e^{2 i \theta}+\bar{a}(z)$ имеем $J=\bar{J}$. Комбинируя полученные ранее интегральные уравнения (3.38) и (3.39), получаем

$$
\begin{aligned}
& \left(\begin{array}{c}
N_{1}(x, z) \\
N_{2}(x, z)
\end{array}\right)=\left(\begin{array}{c}
-i q_{+} \\
z
\end{array}\right)+\sum_{j=1}^{J} \frac{z \bar{b}\left(\bar{z}_{j}\right) e^{-i\left(\bar{z}_{j}-q_{0}^{2} / \bar{z}_{j}\right) x}}{\left(z-\bar{z}_{j}\right) \bar{z}_{j} \bar{a}^{\prime}\left(\bar{z}_{j}\right)} \times \\
& \times\left(\begin{array}{c}
\bar{z}_{j}+\sum_{l=1}^{J} \frac{\bar{z}_{j} b\left(z_{l}\right) e^{i\left(z_{l}-q_{0}^{2} / z_{l}\right) x}}{\left(\bar{z}_{j}-z_{l}\right) z_{l} a^{\prime}\left(z_{l}\right)} N_{1}\left(x, z_{l}\right)+\frac{\bar{z}_{j}}{2 \pi i} \int_{-\infty}^{+\infty} \frac{\rho(\xi)}{\xi\left(\xi-\bar{z}_{j}\right)} e^{i\left(\xi-q_{0}^{2} / \xi\right) x} N_{1}(x, \xi) d \xi \\
i q_{-}+\sum_{l=1}^{J} \frac{\bar{z}_{j} b\left(z_{l}\right) e^{i\left(z_{l}-q_{0}^{2} / z_{l}\right) x}}{\left(\bar{z}_{j}-z_{l}\right) z_{l} a^{\prime}\left(z_{l}\right)} N_{2}\left(x, z_{l}\right)+\frac{\bar{z}_{j}}{2 \pi i} \int_{-\infty}^{+\infty} \frac{\rho(\xi)}{\xi\left(\xi-\bar{z}_{j}\right)} e^{i\left(\xi-q_{0}^{2} / \xi\right) x} N_{2}(x, \xi) d \xi
\end{array}\right)- \\
& -\frac{z}{2 \pi i} \int_{-\infty}^{+\infty} \frac{\bar{\rho}(\xi) d \xi}{\xi(\xi-z)} e^{-i\left(\xi-q_{0}^{2} / \xi\right) x} \times \\
& \times\left(\begin{array}{c}
\xi+\sum_{l=1}^{J} \frac{\xi b\left(z_{l}\right) e^{i\left(z_{l}-q_{0}^{2} / z_{l}\right) x}}{\left(\xi-z_{l}\right) z_{l} a^{\prime}\left(z_{l}\right)} N_{1}\left(x, z_{l}\right)+\frac{\xi}{2 \pi i} \int_{-\infty}^{+\infty} \frac{\rho(\eta)}{\eta(\eta-\xi)} e^{i\left(\eta-q_{0}^{2} / \eta\right) x} N_{1}(x, \eta) d \eta \\
i q_{-}+\sum_{l=1}^{J} \frac{\xi b\left(z_{l}\right) e^{i\left(z_{l}-q_{0}^{2} / z_{l}\right) x}}{\left(\xi-z_{l}\right) z_{l} a^{\prime}\left(z_{l}\right)} N_{2}\left(x, z_{l}\right)+\frac{\xi}{2 \pi i} \int_{-\infty}^{+\infty} \frac{\rho(\eta)}{\eta(\eta-\xi)} e^{i\left(\eta-q_{0}^{2} / \eta\right) x} N_{2}(x, \eta) d \eta
\end{array}\right)
\end{aligned}
$$

Потенциал (3.48) можно восстановить из решения интегрального уравнения (3.49). Можно получить аналогичное уравнение для $\bar{M}(x, z)$ [27].

3.11. Формула следа. Мы показали, что функции $a(z)$ и $\bar{a}(z)$ аналитичны в верхней и нижней полуплоскостях $z$ соответственно. Как упомянуто выше, мы считаем, что $a(z)$ имеет простые нули, которые мы обозначили $z_{j}$. Из условия симметрии $a\left(q_{0}^{2} / z\right)=e^{2 i \theta_{+}} \bar{a}(z)$ получим, что $\bar{a}(z)$ имеет простые нули $q_{0}^{2} / z_{j}$. Введем функции

$$
\gamma(z)=a(z) \prod_{j=1}^{J} \frac{z-q_{0}^{2} / z_{j}}{z-z_{j}}, \quad \bar{\gamma}(z)=\bar{a}(z) \prod_{j=1}^{J} \frac{z-z_{j}}{z-q_{0}^{2} / z_{j}} .
$$


Функции $\gamma(z)$ и $\bar{\gamma}(z)$ аналитичны в верхней и нижней полуплоскостях $z$ соответственно и не имеют нулей в соответствующих им полуплоскостях. Имеем

$$
\begin{array}{llll}
\ln \gamma(z)=\frac{1}{2 \pi i} \int_{-\infty}^{+\infty} \frac{\ln \gamma(\xi)}{\xi-z} d \xi, & \frac{1}{2 \pi i} \int_{-\infty}^{+\infty} \frac{\ln \bar{\gamma}(\xi)}{\xi-z} d \xi=0, & \operatorname{Im} z>0, \\
\ln \bar{\gamma}(z)=-\frac{1}{2 \pi i} \int_{-\infty}^{+\infty} \frac{\ln \bar{\gamma}(\xi)}{\xi-z} d \xi, & \frac{1}{2 \pi i} \int_{-\infty}^{+\infty} \frac{\ln \gamma(\xi)}{\xi-z} d \xi=0, & \operatorname{Im} z<0 .
\end{array}
$$

Складывая или вычитая эти уравнения в каждой полуплоскости соответственно, получим

$$
\begin{array}{ll}
\ln \gamma(z)=\frac{1}{2 \pi i} \int_{-\infty}^{+\infty} \frac{\ln \gamma(\xi) \bar{\gamma}(\xi)}{\xi-z} d \xi, & \operatorname{Im} z>0, \\
\ln \bar{\gamma}(z)=-\frac{1}{2 \pi i} \int_{-\infty}^{+\infty} \frac{\ln \gamma(\xi) \bar{\gamma}(\xi)}{\xi-z} d \xi, & \operatorname{Im} z<0 .
\end{array}
$$

Заметим, что $\gamma(z) \bar{\gamma}(z)=a(z) \bar{a}(z)$, из условия унитарности $a(z) \bar{a}(z)-b(z) \bar{b}(z)=1$ и симметрии $b(z)=\bar{b}(z)$ имеем

$$
\begin{array}{ll}
\ln a(z)=\ln \left(\prod_{j=1}^{J} \frac{z-z_{j}}{z-q_{0}^{2} / z_{j}}\right)+\frac{1}{2 \pi i} \int_{-\infty}^{+\infty} \frac{\ln \left(1+b^{2}(\xi)\right)}{\xi-z} d \xi, & \operatorname{Im} z>0, \\
\ln \bar{a}(z)=\ln \left(\prod_{j=1}^{J} \frac{z-q_{0}^{2} / z_{j}}{z-z_{j}}\right)-\frac{1}{2 \pi i} \int_{-\infty}^{+\infty} \frac{\ln \left(1+b^{2}(\xi)\right)}{\xi-z} d \xi, & \operatorname{Im} z<0 .
\end{array}
$$

Таким образом, мы можем восстановить $a(k), \bar{a}(k)$ через собственные значения (нули) и только одну функцию $b(k)$. Поскольку $a(z) \sim e^{2 i \theta_{+}}$при $z \rightarrow 0$, из формулы следа при $b(\xi)=0$ на вещественной оси получим следующее ограничение для безотражательных потенциалов:

$$
\prod_{j=1}^{J} z_{j}^{2}=q_{0}^{2 J} e^{2 i \theta_{+}} .
$$

3.12. Дискретные данные рассеяния и их симметрии. Чтобы найти безотражательные потенциалы (солитоны), нужно вычислить соответствующие данные рассеяния: $b\left(z_{j}\right)$ и $\bar{b}\left(\bar{z}_{j}\right), a^{\prime}\left(z_{j}\right)$ и $\bar{a}^{\prime}\left(z_{j}\right), j=1,2, \ldots, J$. Последние функции можно вычислить с помощью формулы следа. Поэтому сосредоточимся на вычислении первых. Поскольку

$$
\begin{array}{r}
N_{1}(x, z)=-M_{2}(-x, z), \quad N_{2}(x, z)=M_{1}(-x, z), \\
M_{1}\left(x, z_{j}\right)=b\left(z_{j}\right) e^{i\left(z_{j}-q_{0}^{2} / z_{j}\right) x} N_{1}\left(x, z_{j}\right), \\
\\
M_{2}\left(x, z_{j}\right)=b\left(z_{j}\right) e^{i\left(z_{j}-q_{0}^{2} / z_{j}\right) x} N_{2}\left(x, z_{j}\right),
\end{array}
$$

имеем

$$
\begin{aligned}
& N_{1}\left(x, z_{j}\right)=-b\left(z_{j}\right) e^{-i\left(z_{j}-q_{0}^{2} / z_{j}\right) x} N_{2}\left(-x, z_{j}\right), \\
& N_{2}\left(x, z_{j}\right)=b\left(z_{j}\right) e^{-\left(z_{j}-q_{0}^{2} / z_{j}\right) x} N_{1}\left(-x, z_{j}\right) .
\end{aligned}
$$

Перепишем соотношение (3.57) в виде

$$
N_{2}\left(-x, z_{j}\right)=b\left(z_{j}\right) e^{\left(z_{j}-q_{0}^{2} / z_{j}\right) x} N_{1}\left(x, z_{j}\right) .
$$


Комбинируя (3.58) с (3.56), можно получить следующее условие симметрии для дискретных данных $b\left(z_{j}\right)$ :

$$
-b^{2}\left(z_{j}\right)=1 \text {. }
$$

Подобный анализ показывает, что $\bar{b}\left(\bar{z}_{j}\right)$ удовлетворяет аналогичному уравнению $-\bar{b}^{2}\left(\bar{z}_{j}\right)=1$, т. е.

$$
b\left(z_{j}\right)= \pm i, \quad \bar{b}\left(\bar{z}_{j}\right)= \pm i .
$$

Из условия симметрии $\bar{b}(z)=b(z)$ имеем $\bar{b}\left(z_{j}\right)=b\left(z_{j}\right), b\left(\bar{z}_{j}\right)=\bar{b}\left(\bar{z}_{j}\right)$.

При $J=1$, предполагая, что $0<\theta_{+}<\pi$, получим $z_{1}=q_{0} e^{i \theta_{+}}$. Из формулы следа при $b(\xi)=0$ на вещественной оси вытекают следующие соотношения:

$$
\begin{gathered}
a^{\prime}\left(z_{1}\right)=a^{\prime}\left(q_{0} e^{i \theta_{+}}\right)=\frac{1}{q_{0}\left(e^{i \theta_{+}}-e^{-i \theta_{+}}\right)}, \\
\bar{a}^{\prime}\left(\bar{z}_{1}\right)=a^{\prime}\left(q_{0} e^{-i \theta_{+}}\right)=\frac{1}{q_{0}\left(e^{-i \theta_{+}}-e^{i \theta_{+}}\right)} .
\end{gathered}
$$

Более того, из симметрийной связи $b\left(q_{0}^{2} / z\right)=-\bar{b}(z)$ имеем

$$
\bar{b}\left(q_{0} e^{-i \theta_{+}}\right)=-b\left(q_{0} e^{i \theta_{+}}\right) .
$$

Для удобства запишем $b\left(q_{0} e^{i \theta_{+}}\right)=\delta i$, тогда $\bar{b}\left(q_{0} e^{-i \theta_{+}}\right)=-\delta i$, где $\delta= \pm 1$.

3.13. Эволюция во времени. Поскольку

$$
v_{t}=T v=\left(\begin{array}{cc}
2 i k^{2}+i \sigma q_{0}^{2} & -2 k q_{0} e^{i\left(2 \sigma q_{0}^{2} t+\theta_{ \pm}\right)} \\
-2 \sigma k q_{0} e^{i\left(2 \sigma q_{0}^{2} t+\theta_{ \pm}\right)} & -2 i k^{2}+i \sigma q_{0}^{2}
\end{array}\right) v:=\left(\begin{array}{cc}
A & B \\
C & -A
\end{array}\right) v
$$

где $\sigma=1$, получаем, что как $a(z, t)$, так и $\bar{a}(z, t)$ не зависят от времени, и

$$
\begin{aligned}
& b(z ; t)=b(z ; 0) \exp \left\{-2 i\left[q_{0}^{2}+\frac{1}{2}\left(z^{2}-\frac{q_{0}^{4}}{z^{2}}\right)\right] t\right\}, \\
& \bar{b}(z ; t)=\bar{b}(z ; 0) \exp \left\{2 i\left[q_{0}^{2}+\frac{1}{2}\left(z^{2}-\frac{q_{0}^{4}}{z^{2}}\right)\right] t\right\} .
\end{aligned}
$$

Таким образом,

$$
\begin{aligned}
b\left(q_{0} e^{i \theta_{+}} ; t\right) & =\delta i \exp \left\{-2 i q_{0}^{2}\left[1+i \sin \left(2 \theta_{+}\right)\right] t\right\}, \\
\bar{b}\left(q_{0} e^{-i \theta_{+}} ; t\right) & =-\delta i \exp \left\{2 i q_{0}^{2}\left[1-i \sin \left(2 \theta_{+}\right)\right] t\right\} .
\end{aligned}
$$

3.14. Чистое односолитонное решение. При $J=1$ и $b(\xi, t)=0$ (безотражательный потенциал), решая соответствующую систему уравнений (3.49) (которая является линейной), получим из (3.48)

$$
q(x, t)=q_{0} e^{i\left(2 q_{0}^{2} t+\theta_{+}\right)}\left[1-\frac{2 i \sin \theta_{+} b\left(q_{0} e^{i \theta_{+}} ; t\right) e^{-2 q_{0} x \sin \theta_{+}}}{q_{0} e^{2 i \theta_{+}}} N_{1}\left(x, q_{0} e^{i \theta_{+}} ; t\right)\right],
$$

где

$$
N_{1}\left(x, q_{0} e^{i \theta_{+}}\right)=\frac{\left.-i q_{0} e^{i\left(2 q_{0}^{2} t+\theta_{+}\right.}\right)-q_{0} e^{i \theta_{+}} e^{-2 q_{0} x \sin \theta_{+}} \bar{b}\left(q_{0} e^{-i \theta_{+}} ; t\right)}{1-e^{-4 q_{0} x \sin \theta_{+}} b\left(q_{0} e^{i \theta_{+}} ; t\right) \bar{b}\left(q_{0} e^{-i \theta_{+}} ; t\right)} .
$$




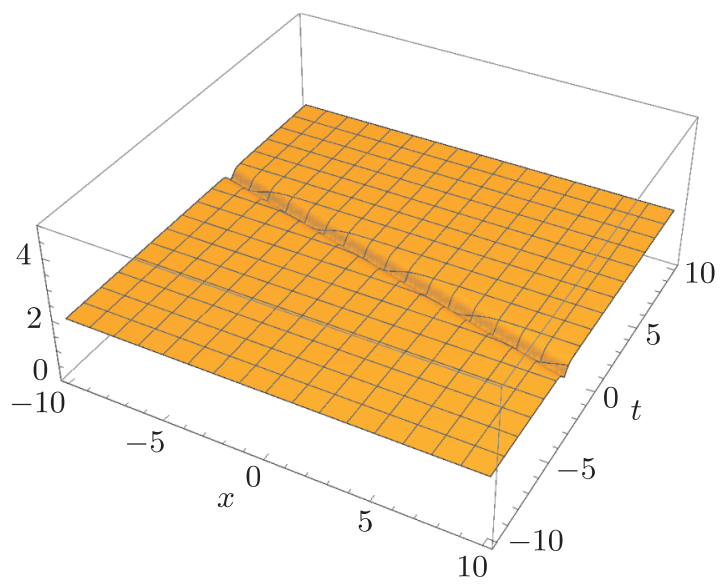

Рис. 1. Амплитуда односолитонного решения $q(x, t)$ как функция $x$ и $t$ при значениях параметров $\sigma=1, \delta=1, \theta_{+}=\pi / 3$ и $q_{0}=2$.

Решая соответствующую дискретную систему (3.49) при $J=1$, получим, что несингулярное чистое односолитонное решение существует только при $\delta=1$, и оно имеет вид

$$
q(x, t)=\frac{1}{2} q_{0} e^{i\left(2 q_{0}^{2} t+\theta_{+}\right)}\left[\left(1+e^{-2 i \theta_{+}}\right)+\left(1-e^{-2 i \theta_{+}}\right) \operatorname{th}\left(q_{0} x \sin \theta_{+}-q_{0}^{2} t \sin \left(2 \theta_{+}\right)\right)\right] .
$$

На рис. 1 показано типичное несингулярное односолитонное решение.

\section{4. НЕЛОКАЛЬНОЕ НУШ С ОБРАЩЕНИЕМ ПРОСТРАНСТВА-ВРЕМЕНИ: $\sigma=1, \theta_{+}+\theta_{-}=\pi$}

4.1. Прямое рассеяние. Рассмотрим ненулевые граничные условия вида (1.6) при $\sigma=1$ и $\theta_{+}+\theta_{-}=\pi$. При таких условиях уравнение $(2.3)$ удобно свести к виду

$$
\frac{\partial^{2} v_{j}}{\partial x^{2}}=-\left(k^{2}+q_{0}^{2}\right) v_{j}, \quad j=1,2 .
$$

Каждое из двух уравнений имеет два линейно независимых решения $e^{i \lambda x}$ и $e^{-i \lambda x}$ при $|x| \rightarrow \infty$, где введены локальные полярные координаты

$$
k-i q_{0}=r_{1} e^{i \theta_{1}}, \quad-\frac{\pi}{2} \leqslant \theta_{1}<\frac{3 \pi}{2}, \quad k+i q_{0}=r_{2} e^{i \theta_{2}}, \quad-\frac{\pi}{2} \leqslant \theta_{2}<\frac{3 \pi}{2},
$$

где $r_{1}=\left|k-i q_{0}\right|$ и $r_{2}=\left|k+i q_{0}\right|$. Можно написать $\lambda(k)=\left(r_{1} r_{2}\right)^{1 / 2} e^{i\left(\theta_{1}+\theta_{2}\right) / 2+i m \pi}$, при этом $m=0,1$ соответственно на слоях I $\left(\mathbb{K}_{1}\right)$ и II $\left(\mathbb{K}_{2}\right)$. Тогда переменную $k$ можно считать принадлежащей поверхности Римана $\mathbb{K}$, состоящей из листов I и II, оба из которых сходятся в разрезе комплексной плоскости вдоль $\Sigma:=\left[-i q_{0}, i q_{0}\right]$, при этом края склеиваются таким образом, что функция $\lambda(k)$ непрерывна на разрезе. Вдоль вещественной оси $k$ имеем $\lambda(k)= \pm \operatorname{sign}(k) \sqrt{k^{2}+q_{0}^{2}}$, где знаки плюс и минус относятся соответственно к листам I и II поверхности Римана, а знак квадратного корня означает главную ветвь вещественнозначной функции квадратного 
корня. Обозначим через $\mathbb{C}^{+}$и $\mathbb{C}^{-}$открытые верхнюю и нижнюю соответственно комплексные полуплоскости, а через $\mathbb{K}^{+}$и $\mathbb{K}^{-}$- открытые верхнюю и нижнюю соответственно комплексные полуплоскости с разрезом вдоль $\Sigma$. Тогда $\lambda$ обеспечивает взаимно однозначное соответствие между следующими множествами:

1) $k \in \mathbb{K}^{+}=\mathbb{C}^{+} \backslash\left(0, i q_{0}\right]$ и $\lambda \in \mathbb{C}^{+}$;

2) $k \in \partial \mathbb{K}^{+}=\mathbb{R} \cup\left\{i s-0^{+}: 0<s<q_{0}\right\} \cup\left\{i q_{0}\right\} \cup\left\{i s+0^{+}: 0<s<q_{0}\right\}$ и $\lambda \in \mathbb{R}$;

3) $k \in \mathbb{K}^{-}=\mathbb{C}^{-} \backslash\left[-i q_{0}, 0\right)$ и $\lambda \in \mathbb{C}^{-}$;

4) $k \in \partial \mathbb{K}^{-}=\mathbb{R} \cup\left\{i s-0^{+}:-q_{0}<s<0\right\} \cup\left\{-i q_{0}\right\} \cup\left\{i s+0^{+}:-q_{0}<s<0\right\}$ и $\lambda \in \mathbb{R}$.

Кроме того, $\lambda^{+}(k)$ и $\lambda^{-}(k)$ означают граничные значения, принимаемые функцией $\lambda(k)$ при $k \in \Sigma$ на соответственно правом и левом краях разреза, при этом $\lambda^{ \pm}(k)= \pm \sqrt{q_{0}^{2}-|k|^{2}}, k=i s \pm 0^{+},|s|<q_{0}$ на левом и правом краях разреза (см. [26], стр. 30, рис. 5, 6).

4.2. Собственные функции. Как и в разделе 3, введем собственные функции $\phi(x, k), \bar{\phi}(x, k), \psi(x, k), \bar{\psi}(x, k)$, задаваемые их граничными условиями. Подставим их в уравнение (2.3) с $\sigma=1$ и получим по формулам (3.5), (3.6)

$$
w=\left(\begin{array}{c}
\lambda+k \\
i q_{+}
\end{array}\right), \quad \bar{w}=\left(\begin{array}{c}
-i q_{-} \\
\lambda+k
\end{array}\right), \quad v=\left(\begin{array}{c}
-i q_{+} \\
\lambda+k
\end{array}\right), \quad \bar{v}=\left(\begin{array}{c}
\lambda+k \\
i q_{-}
\end{array}\right),
$$

где $\lambda=\sqrt{k^{2}+q_{0}^{2}}$. Рассмотрим также функции с постоянными граничными условиями и определим те же ограниченные собственные функции $M(x, k), N(x, k), \bar{M}(x, k)$, $\bar{N}(x, k)$, задаваемые формулами $(3.7)$, с новым определением $\lambda$.

Кроме того, ограниченные собственные функции можно представить с помощью интегральных уравнений, формулы совпадают с формулами (3.8), но данное выше определение $\lambda$ другое.

Используя тот же метод, что и в предыдущем случае $\left(\theta_{+}+\theta_{-}=0\right)$, получим следующий результат.

Теорема 3. Пусть элементы $Q-Q_{ \pm}$принадлежсат $L^{1,1}(\mathbb{R})$, тогда для любого $x \in \mathbb{R}$ собственные функции $M(x, k)$ и $N(x, k)$ непрерывны при $k \in \overline{\mathbb{K}^{+}} \cup \partial \overline{\mathbb{K}^{-}}$ и аналитичны при $k \in \mathbb{K}^{+}, \bar{M}(x, k)$ и $\bar{N}(x, k)$ непрерывны при $k \in \overline{\mathbb{K}^{-}} \cup \partial \overline{\mathbb{K}^{+}}$и аналитичны при $k \in \mathbb{K}^{-}$.

В доказательстве используются ряды Неймана; оно похоже на доказательство, приведенное в работе [26].

\subsection{1. Данные рассеяния. Имеем соотношения}

$$
\phi(x, k)=b(k) \psi(x, k)+a(k) \bar{\psi}(x, k), \quad \bar{\phi}(x, k)=\bar{a}(k) \psi(x, k)+\bar{b}(k) \bar{\psi}(x, k),
$$

справедливые при любом $k$ таком, что все четыре собственные функции существуют. Кроме того,

$$
a(k) \bar{a}(k)-b(k) \bar{b}(k)=1,
$$

где $a(k), \bar{a}(k), b(k)$ и $\bar{b}(k)$ заданы формулами (3.19), но данное в этом разделе определение $\lambda$ отличается от определения, данного в разделе 3 . 
При $k \in\left(-i q_{0}, i q_{0}\right)$ приведенные выше данные рассеяния и собственные функции определены с помощью соответствующих значений на правом и левом краях разреза и помечены верхними индексами \pm , что поясняется ниже. В явном виде имеем для $k \in\left(-i q_{0}, i q_{0}\right)$

$$
\begin{array}{ll}
a^{ \pm}(k)=\frac{W\left(\phi^{ \pm}(x, k), \psi^{ \pm}(x, k)\right)}{2 \lambda^{ \pm}\left(\lambda^{ \pm}+k\right)}, & \bar{a}^{ \pm}(k)=-\frac{W\left(\bar{\phi}^{ \pm}(x, k), \bar{\psi}^{ \pm}(x, k)\right)}{2 \lambda^{ \pm}\left(\lambda^{ \pm}+k\right)} \\
b^{ \pm}(k)=-\frac{W\left(\phi^{ \pm}(x, k), \bar{\psi}^{ \pm}(x, k)\right)}{2 \lambda^{ \pm}\left(\lambda^{ \pm}+k\right)}, & \bar{b}^{ \pm}(k)=\frac{W\left(\bar{\phi}^{ \pm}(x, k), \psi^{ \pm}(x, k)\right)}{2 \lambda^{ \pm}\left(\lambda^{ \pm}+k\right)} .
\end{array}
$$

Тогда из аналитического поведения собственных функций получим следующую теорему.

ТЕорема 4. Предположим, что элементы $Q-Q_{ \pm}$принадлежат $L^{1,1}(\mathbb{R})$, тогда бункция а $(k)$ непрерывна при $k \in \overline{\mathbb{K}^{+}} \cup \partial \overline{\mathbb{K}^{-}} \backslash\left\{ \pm i q_{0}\right\}$ и аналитична при $k \in \mathbb{K}^{+}$, бункция $\bar{a}(k)$ непрерьвна при $k \in \overline{\mathbb{K}^{-}} \cup \partial \overline{\mathbb{K}^{+}} \backslash\left\{ \pm i q_{0}\right\}$ и аналитична при $k \in \mathbb{K}^{-}$. Кроме того, функции $b(k)$ и $\bar{b}(k)$ непрерьвнь при $k \in \mathbb{R} \cup\left(-i q_{0}, i q_{0}\right)$. Если, кроме того, элементы $Q-Q_{ \pm}$растут не быстрее, чем $e^{-a x^{2}}$, где а - вещественное положительное число, то $a(k) \lambda(k), \bar{a}(k) \lambda(k), b(k) \lambda(k)$ и $\bar{b}(k) \lambda(k)$ аналитичны при $k \in \mathbb{K}$.

В доказательстве используются ряды Неймана, оно аналогично доказательству, приведенному в работе [26].

4.3. Симметрийные редукции. Симметрия потенциала порождает симметрию между собственными функциями. Действительно, если функция $v(x, k)=$ $\left(v_{1}(x, k), v_{2}(x, k)\right)^{\mathrm{T}}$ - решение уравнения $(2.1)$ при $\sigma=1$, то $\left(v_{2}(-x, k),-v_{1}(-x, k)\right)^{\mathrm{T}}$ также является решением уравнения (2.1) при $\sigma=1$. Учитывая граничные условия, получим

$$
\psi(x, k)=\left(\begin{array}{cc}
0 & -1 \\
1 & 0
\end{array}\right) \phi(-x, k), \quad \bar{\psi}(x, k)=\left(\begin{array}{cc}
0 & 1 \\
-1 & 0
\end{array}\right) \bar{\phi}(-x, k) .
$$

Аналогично можно получить симметрийные соотношения между собственными функциями, т. е.

$$
N(x, k)=\left(\begin{array}{cc}
0 & -1 \\
1 & 0
\end{array}\right) M(-x, k), \quad \bar{N}(x, k)=\left(\begin{array}{cc}
0 & 1 \\
-1 & 0
\end{array}\right) \bar{M}(-x, k) .
$$

Из вронскианного представления данных рассеяния и приведенных выше симметрийных соотношений имеем $\bar{b}(k)=b(k)$.

4.4. Униформизующие координаты. Введем аналогичным образом униформизующую переменную $z$, определенную с помощью конформного отображения:

$$
z=z(k)=k+\lambda(k),
$$

где $\lambda=\sqrt{k^{2}+q_{0}^{2}}$, обратное отображение задается формулой $k=k(z)=\left(z-q_{0}^{2} / z\right) / 2$. Тогда $\lambda(z)=\left(z+q_{0}^{2} / z\right) / 2$. Пусть $C_{0}$ - окружность радиуса $q_{0}$ с центром в начале координат на плоскости $z$. Мы видим, что справедливы следующие утверждения. 
1. Точка ветвления на обоих листах отображается в окружность $C_{0}$. В частности, $z\left( \pm i q_{0}\right)= \pm i q_{0}$ от каждого листа, $z\left(0_{\mathrm{I}}^{ \pm}\right)= \pm q_{0}$ и $z\left(0_{\mathrm{II}}^{ \pm}\right)=\mp q_{0}$.

2. Плоскость $\mathbb{K}_{1}$ отображается во внешность $C_{0}, \mathbb{K}_{2}$ отображается во внутренность $C_{0}$. В частности, $z\left(\infty_{\mathrm{I}}\right)=\infty$ и $z\left(\infty_{\mathrm{II}}\right)=0$; первый и второй квадранты плоскости $\mathbb{K}_{1}$ отображаются соответственно в первый и второй квадранты снаружи $C_{0}$; первый и второй квадранты плоскости $\mathbb{K}_{2}$ отображаются соответственно во второй и первой квадранты внутри $C_{0} ; z_{\mathrm{I}} z_{\mathrm{II}}=q_{0}^{2}$.

3. Области на плоскости $k$ такие, что $\operatorname{Im} \lambda>0$ и $\operatorname{Im} \lambda<0$, отображаются в $D^{+}=$ $\left\{z \in \mathbb{C}:\left(|z|^{2}-q_{0}^{2}\right) \cdot \operatorname{Im} z>0\right\}$ и $D^{-}=\left\{z \in \mathbb{C}:\left(|z|^{2}-q_{0}^{2}\right) \cdot \operatorname{Im} z<0\right\}$ соответственно (см. [26, стр. 36, рис. 11]).

Тогда получим, что собственные функции $M, N$ аналитичны при $z \in D^{+}$, а собственные функции $\bar{M}, \bar{N}$ аналитичны при $z \in D^{-}$.

4.5. Симметрии в униформизующих координатах. Из приведенных выше симметрийных соотношений между собственными функциями имеем

$$
\psi(x, z)=\left(\begin{array}{rr}
0 & -1 \\
1 & 0
\end{array}\right) \phi(-x, z), \quad \bar{\psi}(x, z)=\left(\begin{array}{rr}
0 & 1 \\
-1 & 0
\end{array}\right) \bar{\phi}(-x, z)
$$

Далее, если $z \rightarrow-q_{0}^{2} / z$, то $(k, \lambda) \rightarrow(k,-\lambda)$. Следовательно,

$$
\phi\left(x,-\frac{q_{0}^{2}}{z}\right)=\frac{q_{0}^{2} / z}{i q_{-}} \bar{\phi}(x, z), \quad \psi\left(x,-\frac{q_{0}^{2}}{z}\right)=\frac{-i q_{+}}{z} \bar{\psi}(x, z), \quad z \in D^{-} .
$$

Аналогично получим

$$
\begin{gathered}
N(x, z)=\left(\begin{array}{rr}
0 & -1 \\
1 & 0
\end{array}\right) M(-x, z), \quad \bar{N}(x, z)=\left(\begin{array}{rr}
0 & 1 \\
-1 & 0
\end{array}\right) \bar{M}(-x, z), \\
\bar{b}(z)=b(z) ; \quad a\left(-\frac{q_{0}^{2}}{z}\right)=-e^{2 i \theta_{+}} \bar{a}(z), \quad z \in D^{-} ; \quad b\left(-\frac{q_{0}^{2}}{z}\right)=-\bar{b}(z) .
\end{gathered}
$$

\section{6. Асимптотическое поведение собственных функций и данных рас-} сеяния. Чтобы решить обратную задачу, нужно определить асимптотическое поведение собственных функций и данных рассеяния как при $z \rightarrow \infty$ на плоскости $\mathbb{K}_{1}$, так и при $z \rightarrow 0$ на плоскости $\mathbb{K}_{2}$. Имеем

$$
\begin{gathered}
\bar{N}(x, z) \sim\left(\begin{array}{c}
z \frac{q(x)}{q_{+}} \\
i q_{-}
\end{array}\right), \quad z \rightarrow 0, \\
a(z)=\left\{\begin{array}{ll}
1, & z \rightarrow \infty, \\
-e^{2 i \theta_{+}}, & z \rightarrow 0,
\end{array} \quad \bar{a}(z)= \begin{cases}1, & z \rightarrow \infty, \\
-e^{-2 i \theta_{+}}, & z \rightarrow 0,\end{cases} \right. \\
\lim _{z \rightarrow \infty} z b(z)=0, \quad \lim _{z \rightarrow 0} \frac{b(z)}{z^{2}}=0 .
\end{gathered}
$$




\section{7. Проблема Римана-Гильберта в униформизующих координатах.}

4.7.1. Левосторонняя задача рассеяния. Чтобы учесть поведение собственных функций, условие "скачка" на $\Sigma$, где

$$
\begin{aligned}
\Sigma:=\left(-\infty,-q_{0}\right) & \cup\left(q_{0},+\infty\right) \cup \overrightarrow{\left(q_{0},-q_{0}\right)} \cup\left\{q_{0} e^{i \theta}, \pi \leqslant \theta \leqslant 2 \pi\right\}_{\text {clockwise, upper circle }} \cup \\
& \cup\left\{q_{0} e^{i \theta},-\pi \leqslant \theta \leqslant 0\right\}_{\text {anticlockwise, lower circle }}
\end{aligned}
$$

можно записать в виде

$$
\begin{aligned}
& \frac{M(x, z)}{z a(z)}-\frac{\bar{N}(x, z)}{z}=\rho(z) e^{i\left(z+q_{0}^{2} / z\right) x} \frac{N(x, z)}{z}, \\
& \frac{\bar{M}(x, z)}{z \bar{a}(z)}-\frac{N(x, z)}{z}=\bar{\rho}(z) e^{-i\left(z+q_{0}^{2} / z\right) x} \frac{\bar{N}(x, z)}{z},
\end{aligned}
$$

так что функции будут ограничены на бесконечности, имея при этом дополнительный полюс при $z=0$. Заметим, что $M(x, z) / a(z)$, как функция от $z$, определена на $D^{+}$, где (по предположению) она имеет простые полюсы $z_{j}$, т. е. $a\left(z_{j}\right)=0$, а функция $\bar{M}(x, z) / \bar{a}(z)$ определена на $D^{-}$, где она имеет простые полюсы $\bar{z}_{j}$, т. е. $\bar{a}\left(\bar{z}_{j}\right)=0$. Следовательно,

$$
\begin{aligned}
& M\left(x, z_{j}\right)=b\left(z_{j}\right) e^{i\left(z_{j}+q_{0}^{2} / z_{j}\right) x} N\left(x, z_{j}\right), \\
& \bar{M}\left(x, \bar{z}_{j}\right)=\bar{b}\left(\bar{z}_{j}\right) e^{-i\left(\bar{z}_{j}+q_{0}^{2} / \bar{z}_{j}\right) x} \bar{N}\left(x, \bar{z}_{j}\right) .
\end{aligned}
$$

Теперь, вычитая значения на бесконечности, индуцированный полюс в начале координат и полюсы (предположительно простые) в $D^{+}$и $D^{-}$соответственно при $a\left(z_{j}\right)=0, j=1,2, \ldots, J$, и $\bar{a}\left(\bar{z}_{j}\right), j=1,2, \ldots, \bar{J}$, получим

$$
\begin{aligned}
& {\left[\frac{M(x, z)}{z a(z)}-\left(\begin{array}{l}
1 \\
0
\end{array}\right)-\frac{1}{z}\left(\begin{array}{c}
0 \\
i q_{-}
\end{array}\right)-\sum_{j=1}^{J} \frac{M\left(x, z_{j}\right)}{\left(z-z_{j}\right) z_{j} a^{\prime}\left(z_{j}\right)}\right]-} \\
& -\left[\frac{\bar{N}(x, z)}{z}-\left(\begin{array}{c}
1 \\
0
\end{array}\right)-\frac{1}{z}\left(\begin{array}{c}
0 \\
i q_{-}
\end{array}\right)-\sum_{j=1}^{J} \frac{b\left(z_{j}\right) e^{i\left(z_{j}+q_{0}^{2} / z_{j}\right) x} N\left(x, z_{j}\right)}{\left(z-z_{j}\right) z_{j} a^{\prime}\left(z_{j}\right)}\right]= \\
& =\rho(z) e^{i\left(z+q_{0}^{2} / z\right) x} \frac{N(x, z)}{z}, \\
& {\left[\frac{\bar{M}(x, z)}{z \bar{a}(z)}-\left(\begin{array}{l}
0 \\
1
\end{array}\right)-\frac{1}{z}\left(\begin{array}{c}
-i q_{+} \\
0
\end{array}\right)-\sum_{j=1}^{\bar{J}} \frac{\bar{M}\left(x, \bar{z}_{j}\right)}{\left(z-\bar{z}_{j}\right) \bar{z}_{j} a^{\prime}\left(\bar{z}_{j}\right)}\right]-} \\
& -\left[\frac{N(x, z)}{z}-\left(\begin{array}{l}
0 \\
1
\end{array}\right)-\frac{1}{z}\left(\begin{array}{c}
-i q_{+} \\
0
\end{array}\right)-\sum_{j=1}^{\bar{J}} \frac{\bar{b}\left(\bar{z}_{j}\right) e^{-i\left(\bar{z}_{j}+q_{0}^{2} / \bar{z}_{j}\right) x} \bar{N}\left(x, \bar{z}_{j}\right)}{\left(z-\bar{z}_{j}\right) \bar{z}_{j} \bar{a}^{\prime}\left(\bar{z}_{j}\right)}\right]= \\
& =\bar{\rho}(z) e^{-i\left(z+q_{0}^{2} / z\right) x} \frac{\bar{N}(x, z)}{z} .
\end{aligned}
$$

Введем теперь проекторы

$$
P_{ \pm}(f)(z)=\frac{1}{2 \pi i} \int_{\Sigma} \frac{f(\xi)}{\xi-(z \pm i 0)} d \xi
$$


где $z$ принадлежит верхней и нижней полуплоскостям и

$$
\begin{aligned}
\Sigma:=\left(-\infty,-q_{0}\right) & \cup\left(q_{0},+\infty\right) \cup \overrightarrow{\left(q_{0},-q_{0}\right)} \cup\left\{q_{0} e^{i \theta}, \pi \leqslant \theta \leqslant 2 \pi\right\}_{\text {clockwise } \text {,upper circle }} \cup \\
& \cup\left\{q_{0} e^{i \theta},-\pi \leqslant \theta \leqslant 0\right\}_{\text {anticlockwise,lower circle }}
\end{aligned}
$$

Если функции $f_{ \pm}(\xi)$ аналитичны в $D^{ \pm}$и убывают при больших $\xi$, то

$$
P_{ \pm}\left(f_{ \pm}\right)(z)= \pm f_{ \pm}(z), \quad P_{\mp}\left(f_{ \pm}\right)(z)=0 .
$$

Применяя операторы $P_{-}$к (4.17) и $P_{+}$к $(4.18)$, получаем

$$
\begin{aligned}
& \bar{N}(x, z)=\left(\begin{array}{c}
z \\
i q_{-}
\end{array}\right)+\sum_{j=1}^{J} \frac{z b\left(z_{j}\right) e^{i\left(z_{j}+q_{0}^{2} / z_{j}\right) x} N\left(x, z_{j}\right)}{\left(z-z_{j}\right) z_{j} a^{\prime}\left(z_{j}\right)}+ \\
&+\frac{z}{2 \pi i} \int_{\Sigma} \frac{\rho(\xi)}{\xi(\xi-z)} e^{i\left(\xi+q_{0}^{2} / \xi\right) x} N(x, \xi) d \xi \\
& N(x, z)=\left(\begin{array}{c}
-i q_{+} \\
z
\end{array}\right)+\sum_{j=1} \frac{z \bar{b}\left(\bar{z}_{j}\right) e^{-i\left(\bar{z}_{j}+q_{0}^{2} / \bar{z}_{j}\right) x} \bar{N}\left(x, \bar{z}_{j}\right)}{\left(z-\bar{z}_{j}\right) \bar{z}_{j} \bar{a}^{\prime}\left(\bar{z}_{j}\right)}- \\
&-\frac{z}{2 \pi i} \int_{\Sigma} \frac{\bar{\rho}(\xi)}{\xi(\xi-z)} e^{-i\left(\xi+q_{0}^{2} / \xi\right) x} \bar{N}(x, \xi) d \xi .
\end{aligned}
$$

Аналогично можно решить правостороннюю задачу рассеяния, которая имеет вид

$$
\begin{aligned}
\bar{M}(x, z)= & \left(\begin{array}{c}
-i q_{-} \\
z
\end{array}\right)+\sum_{j=1}^{J} \frac{-z \bar{b}\left(z_{j}\right) M\left(x, z_{j}\right) e^{-i\left(z_{j}+q_{0}^{2} / z_{j}\right) x}}{\left(z-z_{j}\right) z_{j} a^{\prime}\left(z_{j}\right)}+ \\
& +\frac{z}{2 \pi i} \int_{\Sigma} \frac{\rho^{*}\left(-\xi^{*}\right)}{\xi(\xi-z)} e^{-i\left(\xi+q_{0}^{2} / \xi\right) x} M(x, \xi) d \xi, \\
M(x, z)= & \left(\begin{array}{c}
z \\
i q_{+}
\end{array}\right)+\sum_{j=1}^{\bar{J}} \frac{-z b\left(\bar{z}_{j}\right) \bar{M}\left(x, \bar{z}_{j}\right) e^{i\left(\bar{z}_{j}+q_{0}^{2} / \bar{z}_{j}\right) x}}{\left(z-\bar{z}_{j}\right) \bar{z}_{j} \bar{a}^{\prime}\left(\bar{z}_{j}\right)}- \\
& -\frac{z}{2 \pi i} \int_{\Sigma} \frac{\bar{\rho}^{*}\left(-\xi^{*}\right)}{\xi(\xi-z)} e^{i\left(\xi+q_{0}^{2} / \xi\right) x} \bar{M}(x, \xi) d \xi .
\end{aligned}
$$

4.8. Восстановление потенциалов. Заметим, что $\bar{N}_{1}(x, z) / z \sim q(x) / q_{+}$при $z \rightarrow 0$, и в этом пределе

$$
\frac{\bar{N}_{1}(x, z)}{z} \sim 1+\sum_{j=1}^{J} \frac{b\left(z_{j}\right) e^{i\left(z_{j}+q_{0}^{2} / z_{j}\right) x}}{-z_{j}^{2} a^{\prime}\left(z_{j}\right)} N_{1}\left(x, z_{j}\right)+\frac{1}{2 \pi i} \int_{\Sigma} \frac{\rho(\xi)}{\xi^{2}} e^{i\left(\xi+q_{0}^{2} / \xi\right) x} N_{1}(x, \xi) d \xi
$$

имеем

$$
\begin{aligned}
q(x)=q_{+}[1 & +\sum_{j=1}^{J} \frac{b\left(z_{j}\right) e^{i\left(z_{j}+q_{0}^{2} / z_{j}\right) x}}{-z_{j}^{2} a^{\prime}\left(z_{j}\right)} N_{1}\left(x, z_{j}\right)+ \\
& \left.+\frac{1}{2 \pi i} \int_{\Sigma} \frac{\rho(\xi)}{\xi^{2}} e^{i\left(\xi+q_{0}^{2} / \xi\right) x} N_{1}(x, \xi) d \xi\right]
\end{aligned}
$$


4.9. Замыкание системы. Из равенства $a\left(-q_{0}^{2} / z\right)=-e^{2 i \theta+} \bar{a}(z)$ найдем $J=\bar{J}$. Комбинируя интегральные уравнения для собственных функций, получим

$$
\begin{aligned}
& \left(\begin{array}{c}
N_{1}(x, z) \\
N_{2}(x, z)
\end{array}\right)=\left(\begin{array}{c}
-i q_{+} \\
z
\end{array}\right)+\sum_{j=1}^{J} \frac{z \bar{b}\left(\bar{z}_{j}\right) e^{-i\left(\bar{z}_{j}+q_{0}^{2} / \bar{z}_{j}\right) x}}{\left(z-\bar{z}_{j}\right) \bar{z}_{j} \bar{a}^{\prime}\left(\bar{z}_{j}\right)} \times \\
& \times\left(\begin{array}{c}
\bar{z}_{j}+\sum_{l=1}^{J} \frac{\bar{z}_{j} b\left(z_{l}\right) e^{i\left(z_{l}+q_{0}^{2} / z_{l}\right) x}}{\left(\bar{z}_{j}-z_{l}\right) z_{l} a^{\prime}\left(z_{l}\right)} N_{1}\left(x, z_{l}\right)+\frac{\bar{z}_{j}}{2 \pi i} \int_{\Sigma} \frac{\rho(\xi)}{\xi\left(\xi-\bar{z}_{j}\right)} e^{i\left(\xi+q_{0}^{2} / \xi\right) x} N_{1}(x, \xi) d \xi \\
i q_{-}+\sum_{l=1}^{J} \frac{\bar{z}_{j} b\left(z_{l}\right) e^{i\left(z_{l}+q_{0}^{2} / z_{l}\right) x}}{\left(\bar{z}_{j}-z_{l}\right) z_{l} a^{\prime}\left(z_{l}\right)} N_{2}\left(x, z_{l}\right)+\frac{\bar{z}_{j}}{2 \pi i} \int_{\Sigma} \frac{\rho(\xi)}{\xi\left(\xi-\bar{z}_{j}\right)} e^{i\left(\xi+q_{0}^{2} / \xi\right) x} N_{2}(x, \xi) d \xi
\end{array}\right)- \\
& -\frac{\bar{z}}{2 \pi i} \int_{\Sigma} \frac{\bar{\rho}(\xi)}{\xi(\xi-z)} e^{-i\left(\xi+q_{0}^{2} / \xi\right) x} \times \\
& \times\left(\begin{array}{c}
\xi+\sum_{l=1}^{J} \frac{\xi b\left(z_{l}\right) e^{i\left(z_{l}-q_{0}^{2} / z_{l}\right) x}}{\left(\xi-z_{l}\right) z_{l} a^{\prime}\left(z_{l}\right)} N_{1}\left(x, z_{l}\right)+\frac{\xi}{2 \pi i} \int_{\Sigma} \frac{\rho(\eta)}{\eta(\eta-\xi)} e^{i\left(\eta+q_{0}^{2} / \eta\right) x} N_{1}(x, \eta) d \eta \\
i q_{-}+\sum_{l=1}^{J} \frac{\xi b\left(z_{l}\right) e^{i\left(z_{l}+q_{0}^{2} / z_{l}\right) x}}{\left(\xi-z_{l}\right) z_{l} a^{\prime}\left(z_{l}\right)} N_{2}\left(x, z_{l}\right)+\frac{\xi}{2 \pi i} \int_{\Sigma} \frac{\rho(\eta)}{\eta(\eta-\xi)} e^{i\left(\eta+q_{0}^{2} / \eta\right) x} N_{2}(x, \eta) d \eta
\end{array}\right)
\end{aligned}
$$

Из соотношения (4.24) можно восстановить потенциал.

4.10. Формула следа. Как и в разделе 3 , получим формулу следа в следующем виде:

$$
\begin{array}{ll}
\ln a(z)=\ln \left(\prod_{j=1}^{J} \frac{z-z_{j}}{z+q_{0}^{2} / z_{j}}\right)+\frac{1}{2 \pi i} \int_{\Sigma} \frac{\ln \left(1+b^{2}(\xi)\right)}{\xi-z} d \xi, \quad z \in D^{+}, \\
\ln \bar{a}(z)=\ln \left(\prod_{j=1}^{J} \frac{z+q_{0}^{2} / z_{j}}{z-z_{j}}\right)-\frac{1}{2 \pi i} \int_{\Sigma} \frac{\ln \left(1+b^{2}(\xi)\right)}{\xi-z} d \xi, \quad z \in D^{-} .
\end{array}
$$

4.11. Дискретные данные рассеяния и их симметрии. Чтобы найти безотражательные потенциалы и солитоны, нужно уметь вычислять соответствующие дискретные данные рассеяния. Коэффициенты $b\left(z_{j}\right)$ и $\bar{b}\left(\bar{z}_{j}\right), j=1,2, \ldots, J$, можно вычислить так же, как и в разделе 3, имеем

$$
\bar{b}\left(z_{j}\right)=b\left(z_{j}\right)= \pm i, \quad b\left(\bar{z}_{j}\right)=\bar{b}\left(\bar{z}_{j}\right)= \pm i .
$$

Поскольку $a(z) \sim-e^{2 i \theta_{+}}$при $z \rightarrow 0$, из формулы следа при $b(\xi)=0$ на разрезе $\Sigma$ имеем следующее ограничение для безотражательных потенциалов:

$$
\prod_{j=1}^{J} z_{j}= \pm(-1)^{(J+1) / 2} q_{0}^{J} e^{i \theta_{+}} .
$$

Мы утверждаем, что $J \geqslant 2$. Иначе, если $J=1$, получаем $z_{1}= \pm q_{0} e^{i \theta_{+}}$. Это значит, что собственное значение $z_{1}$ лежит на окружности, которая в данном случае является непрерывным спектром. Такие собственные числа нам не подходят, они здесь не рассматриваются. 
4.12. Безотражательные данные рассеяния для двух собственных значений. Рассмотрим данные рассеяния, связанные с двумя собственными значениями, т. е. $J=2$ и отражение отсутствует. Заметим, что $\left|z_{1}\right| \cdot\left|z_{2}\right|=q_{0}^{2}$. Пусть теперь $\pi<\theta_{+}<3 \pi / 2$ и $z_{1}=q_{1} e^{i \theta_{1}}$, где $q_{1}>q_{0}$ и $0<\theta_{1}<\pi / 2$, тогда $z_{2}=$ $\left(q_{0}^{2} / q_{1}\right) e^{i\left(\theta_{+}-\theta_{1}+\pi / 2\right)}$, где $\pi<\arg z_{2}<2 \pi$. В частности, можно выбрать $z_{1}=i q_{1}$, $z_{2}=-i\left(q_{0}^{2} / q_{1}\right)$ и $\theta_{+}=\pi / 2$, тогда $\bar{z}_{1}=i\left(q_{0}^{2} / q_{1}\right), \bar{z}_{2}=-i q_{1}$ и $z_{1} z_{2}=q_{0}^{2}$. По формуле следа при $b(\xi)=0$ на разрезе $\Sigma$ получим

$$
a(z)=\frac{z-i q_{1}}{z-i\left(q_{0}^{2} / q_{1}\right)} \cdot \frac{z+i\left(q_{0}^{2} / q_{1}\right)}{z+i q_{1}}, \quad \bar{a}(z)=\frac{z-i\left(q_{0}^{2} / q_{1}\right)}{z-i q_{1}} \cdot \frac{z+i q_{1}}{z+i\left(q_{0}^{2} / q_{1}\right)}
$$

Имеем

$$
\begin{aligned}
a^{\prime}\left(i q_{1}\right) & =\frac{-i\left(q_{1}^{2}+q_{0}^{2}\right)}{2 q_{1}\left(q_{1}^{2}-q_{0}^{2}\right)}, & a^{\prime}\left(-i \frac{q_{0}^{2}}{q_{1}}\right) & =\frac{i q_{1}\left(q_{0}^{2}+q_{1}^{2}\right)}{2 q_{0}^{2}\left(q_{0}^{2}-q_{1}^{2}\right)}, \\
\bar{a}^{\prime}\left(i \frac{q_{0}^{2}}{q_{1}}\right) & =-\frac{i q_{1}\left(q_{0}^{2}+q_{1}^{2}\right)}{2 q_{0}^{2}\left(q_{0}^{2}-q_{1}^{2}\right)}, & \bar{a}^{\prime}\left(-i q_{1}\right) & =\frac{i\left(q_{1}^{2}+q_{0}^{2}\right)}{2 q_{1}\left(q_{1}^{2}-q_{0}^{2}\right)} .
\end{aligned}
$$

Более того, из симметрийного соотношения $b\left(-q_{0}^{2} / z\right)=-\bar{b}(z)$, получим

$$
b\left(i q_{1}\right)=\delta_{1} i, \quad b\left(-i \frac{q_{0}^{2}}{q_{1}}\right)=\delta_{2} i, \quad \bar{b}\left(i \frac{q_{0}^{2}}{q_{1}}\right)=-\delta_{1} i, \quad \bar{b}\left(-i q_{1}\right)=-\delta_{2} i .
$$

4.13. Эволюция во времени. Так же, как и в разделе 3 , найдем, что $a(z, t)$ и $\bar{a}(z, t)$ не зависят от времени и

$$
\begin{aligned}
& b(z ; t)=b(z ; 0) \exp \left\{-2 i\left[-q_{0}^{2}+\frac{1}{2}\left(z^{2}-\frac{q_{0}^{4}}{z^{2}}\right)\right] t\right\}, \\
& \bar{b}(z ; t)=\bar{b}(z ; 0) \exp \left\{2 i\left[-q_{0}^{2}+\frac{1}{2}\left(z^{2}-\frac{q_{0}^{4}}{z^{2}}\right)\right] t\right\} .
\end{aligned}
$$

Таким образом,

$$
\begin{aligned}
b\left(i q_{1} ; t\right) & =\delta_{1} i \exp \left\{-2 i\left[-q_{0}^{2}+\frac{1}{2}\left(-q_{1}^{2}+\frac{q_{0}^{4}}{q_{1}^{2}}\right)\right] t\right\}, \\
b\left(-i \frac{q_{0}^{2}}{q_{1}} ; t\right) & =\delta_{2} i \exp \left\{-2 i\left[-q_{0}^{2}+\frac{1}{2}\left(q_{1}^{2}-\frac{q_{0}^{4}}{q_{1}^{2}}\right)\right] t\right\}, \\
\bar{b}\left(i \frac{q_{0}^{2}}{q_{1}} ; t\right) & =-\delta_{1} i \exp \left\{2 i\left[-q_{0}^{2}+\frac{1}{2}\left(q_{1}^{2}-\frac{q_{0}^{4}}{q_{1}^{2}}\right)\right] t\right\}, \\
\bar{b}\left(-i q_{1} ; t\right) & =-\delta_{2} i \exp \left\{2 i\left[-q_{0}^{2}+\frac{1}{2}\left(-q_{1}^{2}+\frac{q_{0}^{4}}{q_{1}^{2}}\right)\right] t\right\} .
\end{aligned}
$$

4.14. Чистое двухсолитонное решение. При $J=2$ и $b(\xi, t)=0$ (безотражательный потенциал), решая соответствующую дискретную систему (4.25), а также из (4.24) получим несингулярное двухсолитонное решение при $\delta_{1} \delta_{2}=-1$. 


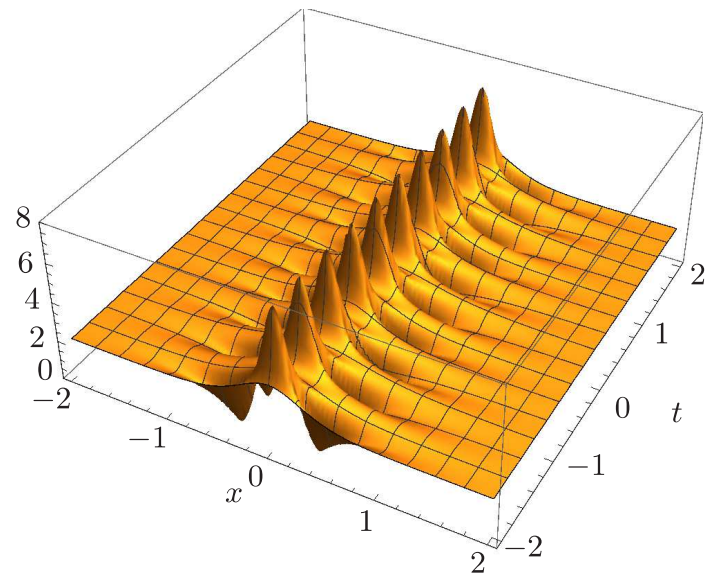

Рис. 2. Амплитуда потенциала $q(x, t)$ при $\delta_{1}=1, \delta_{2}=-1, q_{1}=4$ и $q_{0}=2$.

В этом случае нормировочные постоянные имеют вид

$$
\begin{aligned}
& C_{1}(t):=\frac{b\left(i q_{1} ; t\right)}{a^{\prime}\left(i q_{1} ; t\right)}=-\frac{2 \delta_{1} q_{1}\left(q_{1}^{2}-q_{0}^{2}\right)}{q_{1}^{2}+q_{0}^{2}} \exp \left\{-2 i\left[-q_{0}^{2}+\frac{1}{2}\left(-q_{1}^{2}+\frac{q_{0}^{4}}{q_{1}^{2}}\right)\right] t\right\}, \\
& C_{2}(t):=\frac{b\left(-i\left(q_{0}^{2} / q_{1}\right) ; t\right)}{a^{\prime}\left(-i\left(q_{0}^{2} / q_{1}\right) ; t\right)}=\frac{2 \delta_{2} q_{0}^{2}\left(q_{0}^{2}-q_{1}^{2}\right)}{q_{1}\left(q_{0}^{2}+q_{1}^{2}\right)} \exp \left\{-2 i\left[-q_{0}^{2}+\frac{1}{2}\left(q_{1}^{2}-\frac{q_{0}^{4}}{q_{1}^{2}}\right)\right] t\right\}, \\
& \bar{C}_{1}(t):=\frac{\bar{b}\left(i\left(q_{0}^{2} / q_{1}\right) ; t\right)}{\bar{a}^{\prime}\left(i\left(q_{0}^{2} / q_{1}\right) ; t\right)}=\frac{2 \delta_{1} q_{0}^{2}\left(q_{0}^{2}-q_{1}^{2}\right)}{q_{1}\left(q_{0}^{2}+q_{1}^{2}\right)} \exp \left\{2 i\left[-q_{0}^{2}+\frac{1}{2}\left(q_{1}^{2}-\frac{q_{0}^{4}}{q_{1}^{2}}\right)\right] t\right\}, \\
& \bar{C}_{2}(t):=\frac{\bar{b}\left(-i q_{1} ; t\right)}{\bar{a}^{\prime}\left(-i q_{1} ; t\right)}=-\frac{2 \delta_{2} q_{1}\left(q_{1}^{2}-q_{0}^{2}\right)}{q_{1}^{2}+q_{0}^{2}} \exp \left\{2 i\left[-q_{0}^{2}+\frac{1}{2}\left(-q_{1}^{2}+\frac{q_{0}^{4}}{q_{1}^{2}}\right)\right] t\right\} .
\end{aligned}
$$

При $\delta_{1}=1$ и $\delta_{2}=-1$ получим

$$
\begin{aligned}
& q(x, t)=e^{-2 i q_{0}^{2} t} \times \\
& \times \frac{\left[i\left(q_{0}^{4}+q_{1}^{4}\right) \cos \left(\frac{q_{0}^{4}-q_{1}^{4}}{q_{1}^{2}} t\right)+i q_{0} q_{1}\left(q_{0}^{2}+q_{1}^{2}\right) \operatorname{ch}\left(\frac{q_{0}^{2}-q_{1}^{2}}{q_{1}} x\right)+\left(q_{0}^{4}-q_{1}^{4}\right) \sin \left(\frac{q_{0}^{4}-q_{1}^{4}}{q_{1}^{2}} t\right)\right]}{q_{1}\left[2 q_{0} q_{1} \cos \left(\frac{\left(q_{0}^{4}-q_{1}^{4}\right) t}{q_{1}^{2}}\right)+\left(q_{0}^{2}+q_{1}^{2}\right) \operatorname{ch}\left(\frac{\left(q_{0}^{2}-q_{1}^{2}\right) x}{q_{1}}\right)\right]} .
\end{aligned}
$$

При $\delta_{1}=-1$ и $\delta_{2}=1$ найдем

$$
\begin{aligned}
& q(x, t)=e^{-2 i q_{0}^{2} t} \times \\
& \times \frac{\left[-i\left(q_{0}^{4}+q_{1}^{4}\right) \cos \left(\frac{q_{0}^{4}-q_{1}^{4}}{q_{1}^{2}} t\right)+i q_{0} q_{1}\left(q_{0}^{2}+q_{1}^{2}\right) \operatorname{ch}\left(\frac{q_{0}^{2}-q_{1}^{2}}{q_{1}} x\right)+\left(-q_{0}^{4}+q_{1}^{4}\right) \sin \left(\frac{q_{0}^{4}-q_{1}^{4}}{q_{1}^{2}} t\right)\right]}{q_{1}\left[-2 q_{0} q_{1} \cos \left(\frac{\left(q_{0}^{4}-q_{1}^{4}\right) t}{q_{1}^{2}}\right)+\left(q_{0}^{2}+q_{1}^{2}\right) \operatorname{ch}\left(\frac{\left(q_{0}^{2}-q_{1}^{2}\right) x}{q_{1}}\right)\right]} .
\end{aligned}
$$

На рис. 2 изображено типичное двухсолитонное решение типа бризера при $\delta_{1}=$ $-\delta_{1}=1$. 


\section{5. ПРОСТРАНСТВЕННО-ЗАВИСИМЫЕ ГРАНИЧНЫЕ УСЛОВИЯ}

\section{1. НУШ с обращением пространства-времени: пространственные гра-} ничные условия. Рассмотрим НУШ с обращением пространства-времени

$$
i q_{t}(x, t)=q_{x x}(x, t)-2 \sigma q^{2}(x, t) q(-x,-t)
$$

с граничным условием

$$
q(x, t) \rightarrow q_{0} e^{i\left(\alpha t+\beta x+\theta_{ \pm}\right)}
$$

при $x \rightarrow \pm \infty$, где $q_{0}>0,0 \leqslant \theta_{ \pm}<2 \pi$, а $\alpha$ и $\beta$ - вещественные параметры. Мы видим, что $\alpha=\beta^{2}+2 \sigma \rho q_{0}^{2}$, тогда граничные условия принимают вид

$$
q(x, t) \rightarrow q_{0} e^{i\left[\left(\beta^{2}+2 \sigma \rho q_{0}^{2}\right) t+\beta x+\theta_{ \pm}\right]}
$$

при $x \rightarrow \pm \infty$, где $\beta$ - вещественный параметр, а $\rho= \pm 1$. В частности, $\rho=1$ при $\theta_{+}+\theta_{-}=0, \rho=-1$ при $\theta_{+}+\theta_{-}=\pi$. Положив $q(x, t)=\tilde{q}(x, t) e^{i \beta x}$, имеем

$$
i \tilde{q}_{t}(x, t)=\tilde{q}_{x x}(x, t)+2 i \beta \tilde{q}_{x}(x, t)-\left[\beta^{2}+2 \sigma \tilde{q}(x, t) \tilde{q}(-x,-t)\right] \tilde{q}(x, t),
$$

что связано со следующей совместной $(2 \times 2)$-системой:

$$
v_{x}=X v=\left(\begin{array}{cc}
-i k & \tilde{q}(x, t) \\
\sigma \tilde{q}(-x,-t) & i k
\end{array}\right) v,
$$

$$
\begin{aligned}
& v_{t}=T v= \\
& =\left(\begin{array}{cc}
2 i k^{2}-2 \beta i k+ & -2 k \tilde{q}(x, t)+2 \beta \tilde{q}(x, t)-i \tilde{q}_{x}(x, t) \\
+i \sigma \tilde{q}(x, t) \tilde{q}(-x,-t)+\frac{\beta^{2}}{2} i & -2 i k^{2}+2 \beta i k- \\
-2 \sigma k \tilde{q}(-x,-t)+ & -i \sigma \tilde{q}(x, t) \tilde{q}(-x,-t)-\frac{\beta^{2}}{2} i
\end{array}\right) v .
\end{aligned}
$$

Тогда получим, что $a(k, t)$ и $\bar{a}(k, t)$ не зависят от времени, а $b(k, t)$ и $\bar{b}(k, t)$ зависят от времени следующим образом:

$$
\begin{aligned}
& b(k, t)=b(0) \exp \left\{-2 i\left[\sigma \rho q_{0}^{2}+\frac{\beta^{2}}{2}-2 \lambda(\beta-k)\right] t\right\}, \\
& \bar{b}(k, t)=\bar{b}(0) \exp \left\{2 i\left[\sigma \rho q_{0}^{2}+\frac{\beta^{2}}{2}-2 \lambda(\beta-k)\right] t\right\} .
\end{aligned}
$$

Как обсуждалось в разделе 1 , положим $\sigma=1$, а из раздела 3 возьмем $J=1$ и $\delta=1$. Тогда

$$
\begin{aligned}
b\left(q_{0} e^{i \theta_{+}} ; t\right) & =i \exp \left\{-2 i\left[q_{0}^{2}+\frac{\beta^{2}}{2}-2 i q_{0} \sin \theta_{+}\left(\beta-q_{0} \cos \theta_{+}\right)\right] t\right\}, \\
\bar{b}\left(q_{0} e^{-i \theta_{+}} ; t\right) & =-i \exp \left\{2 i\left[q_{0}^{2}+\frac{\beta^{2}}{2}+2 i q_{0} \sin \theta_{+}\left(\beta-q_{0} \cos \theta_{+}\right)\right] t\right\} .
\end{aligned}
$$

Таким образом, мы нашли чистое односолитонное решение

$$
\begin{aligned}
& q(x, t)=e^{i \beta x} q_{0} e^{2 i q_{0}^{2} t} \times \\
& \times \frac{\left(-e^{-i \theta_{+}}+e^{i \theta_{+}+4 q_{0} \sin \theta_{+}\left(x+2 \beta t-2 q_{0} t \cos \theta_{+}\right)}-2 i \sin \theta_{+} e^{-i \beta^{2} t+2 q_{0} \sin \theta_{+}\left(x+2 \beta t-2 q_{0} t \cos \theta_{+}\right)}\right)}{e^{4 q_{0} \sin \theta_{+}\left(x+2 \beta t-2 q_{0} t \cos \theta_{+}\right)}-1} .
\end{aligned}
$$


ЗАМЕчАниЕ 2 . При $\beta \neq 0$ приведенное выше решение сингулярно вдоль выколотой линии $x=C t$, где $C, t \neq 0$.

5.2. Стандартное НУШ: пространственные граничные условия. НУШ имеет вид

$$
i q_{t}(x, t)=q_{x x}(x, t)-2 \sigma|q(x, t)|^{2} q(x, t),
$$

где $q^{*}$ означает комплексное сопряжение потенциала $q$, а $\sigma=\mp 1$. Рассмотрим граничное условие

$$
q(x, t) \rightarrow q_{0} e^{i\left(\alpha t+\beta x+\theta_{ \pm}\right)}, \quad x \rightarrow \pm \infty,
$$

где $q_{0}>0,0 \leqslant \theta_{ \pm}<2 \pi$, оба параметра $\alpha$ и $\beta$ вещественные. Легко видеть, что $\alpha=\beta^{2}+2 \sigma q_{0}^{2}$, тогда граничное условие принимает вид

$$
q(x, t) \rightarrow q_{0} e^{i\left[\left(\beta^{2}+2 \sigma q_{0}^{2}\right) t+\beta x+\theta_{ \pm}\right]}
$$

при $x \rightarrow \pm \infty$, где $\beta$ - вещественный параметр. Положив $q(x, t)=\tilde{q}(x, t) e^{i \beta x}$, получим уравнение

$$
i \tilde{q}_{t}=\tilde{q}_{x x}+2 i \beta \tilde{q}_{x}-\left(\beta^{2}+2 \sigma|\tilde{q}|^{2}\right) \tilde{q},
$$

которое связано с совместной $(2 \times 2)$-системой

$$
\begin{gathered}
v_{x}=X v=\left(\begin{array}{cc}
-i k & \tilde{q}(x, t) \\
\sigma \tilde{q}^{*}(x, t) & i k
\end{array}\right) v \\
v_{t}=T v=\left(\begin{array}{cc}
2 i k^{2}-2 \beta i k+i \sigma|\tilde{q}|^{2}+\frac{\beta^{2}}{2} i & -2 \tilde{q} k+2 \beta \tilde{q}-i \tilde{q}_{x} \\
-2 \sigma \tilde{q}^{*} k+i \sigma \tilde{q}_{x}^{*}+2 \beta \sigma \tilde{q}^{*} & -2 i k^{2}+2 \beta i k-i \sigma|\tilde{q}|^{2}-\frac{\beta^{2}}{2} i
\end{array}\right) v .
\end{gathered}
$$

Тогда получим, что как $a(k, t)$, так и $\bar{a}(k, t)$ не зависят от времени,

$$
\begin{aligned}
& b(k, t)=b(0) \exp \left\{-2 i\left[\sigma q_{0}^{2}+\frac{\beta^{2}}{2}-2 \lambda(\beta-k)\right] t\right\}, \\
& \bar{b}(k, t)=\bar{b}(0) \exp \left\{2 i\left[\sigma q_{0}^{2}+\frac{\beta^{2}}{2}-2 \lambda(\beta-k)\right] t\right\} .
\end{aligned}
$$

Задача рассеяния (5.13) та же самая, что и для НУШ с ненулевым граничным условием

$$
\tilde{q}(x, t) \rightarrow q_{0} e^{i\left(2 \sigma q_{0}^{2} t+\theta_{ \pm}\right)}, \quad x \rightarrow \pm \infty .
$$

Единственное отличие в формулировках МОЗР для стандартного НУШ и уравнения (5.12) заключается в эволюции во времени. На основе задачи рассеяния и односолитонного решения дефокусирующего НУШ [15], получим при $\sigma=1$

$$
\begin{aligned}
q(x, t)=\tilde{q} e^{i \beta x}=e^{i \beta x}\left[q_{0} e^{i\left[\left(\beta^{2}+2 q_{0}^{2}\right) t+\theta_{+}\right]}+\right. \\
\\
\quad+\frac{i C_{1}^{*}(0) \alpha_{1}^{*} \exp \left[2 i\left(q_{0}^{2}+\beta^{2} / 2\right) t-4 v_{1}\left(\beta-k_{1}\right) t-2 v_{1} x\right]}{\left.1+\left(q_{0}\left|C_{1}(0)\right| / 2 v_{1}\right) e^{-2 v_{1} x-4 v_{1}\left(\beta-k_{1}\right) t}\right]}
\end{aligned}
$$


где $\alpha_{1}=k_{1}+i v_{1}, v_{1}=\sqrt{q_{0}^{2}-k_{1}^{2}},-q_{0}<k_{1}<q_{0}, e^{2 v_{1} x_{0}}=q_{0}\left|C_{1}(0)\right| / 2 v_{1}$ и $C_{1}^{*}(0)=$ $-\left|C_{1}(0)\right| e^{i \theta_{+}}$. Это выражение можно переписать в виде

$$
q(x, t)=q_{0} e^{2 i q_{0}^{2} t} e^{i\left(\beta x+\beta^{2} t\right)}\left[e^{i \theta_{+}}+\frac{i C_{1}^{*}(0)\left(\alpha_{1}^{*} / q_{0}\right) e^{-2 v_{1}\left(x+2 \beta t-2 k_{1} t\right)}}{1+\left(q_{0}\left|C_{1}(0)\right| / 2 v_{1}\right) e^{-2 v_{1}\left(x+2 \beta t-2 k_{1} t\right)}}\right] .
$$

Одним из свойств НУШ является галилеева инвариантность, т. е. если $q_{1}(x, t)$ является решением НУШ и удовлетворяет граничному условию $q_{1}(x, t) \rightarrow q_{0} e^{i\left(2 \sigma q_{0}^{2} t+\theta_{ \pm}\right)}$ при $x \rightarrow \pm \infty$, то $q_{2}(x, t):=q_{1}(x+2 \beta t, t) e^{i\left(\beta x+\beta^{2} t\right)}$ также является решением НУШ и удовлетворяет граничному условию $q_{2}(x, t) \rightarrow q_{0} e^{i\left[\left(\beta^{2}+2 \sigma q_{0}^{2}\right) t+\beta x+\theta_{ \pm}\right]}$при $x \rightarrow \pm \infty$. Если $\sigma=1$, односолитонное решение принимает вид

$$
q_{1}(x, t)=q_{0} e^{2 i q_{0}^{2} t}\left[e^{i \theta_{+}}+\frac{i C_{1}^{*}(0)\left(\alpha_{1}^{*} / q_{0}\right) e^{-2 v_{1}\left(x-2 k_{1} t\right)}}{1+\left(q_{0}\left|C_{1}(0)\right| / 2 v_{1}\right) e^{-2 v_{1}\left(x-2 k_{1} t\right)}}\right]
$$

Имеем $q(x, t)=q_{1}(x+2 \beta t, t) e^{i\left(\beta x+\beta^{2} t\right)}$, а это означает, что решение ОЗР согласуется с решением, основанным на галилеевой инвариантности НУШ. Полагая $\alpha=k_{1} / q_{0}$ и $\gamma=-v_{1} / q_{0}$, можно упростить (возможно, с точностью до фазы) $q_{1}(x, t)$ и $q(x, t)$ следующим образом:

$$
\begin{aligned}
q_{1}(x, t) & =q_{0} e^{2 i q_{0}^{2} t}\left[\gamma \operatorname{th}\left(q_{0} \gamma\left(x-2 q_{0} \alpha t-x_{0}\right)\right)-i \alpha\right], \\
q(x, t) & =q_{0} e^{2 i q_{0}^{2} t} e^{i\left(\beta x+\beta^{2} t\right)}\left[\gamma \operatorname{th}\left(q_{0} \gamma\left(x+2 \beta t-2 q_{0} \alpha t-x_{0}\right)\right)-i \alpha\right],
\end{aligned}
$$

где $\alpha^{2}+\gamma^{2}=1$.

Если игнорировать нелинейный член, то линейное уравнение в частных производных

$$
i q_{t}(x, t)-q_{x x}(x, t)=0
$$

также удовлетворяет условию галилеевой инвариантности. Действительно, согласно преобразованию Фурье, функция

$$
u_{1}(x, t)=\frac{1}{2 \pi} \int_{-\infty}^{\infty} b^{(1)}(\xi) e^{i\left(\xi x+\xi^{2} t\right)} d \xi
$$

является решением уравнения (5.21). Положим $u_{2}(x, t)=u_{1}(x+2 \beta t, t) e^{i\left(\beta x+\beta^{2} t\right)}$, тогда

$$
u_{2}(x, t)=\frac{1}{2 \pi} \int_{-\infty}^{\infty} b^{(1)}(\xi) e^{i(\xi+\beta) x} e^{i(\xi+\beta)^{2} t} d \xi
$$

Переопределив переменные $\left(\xi^{\prime}=\xi+\beta\right)$, мы видим, что $u_{2}(x, t)$ также является решением уравнения (5.21), а это означает, что галилеева инвариантность справедлива и для линейной задачи.

Благодарности. Авторы выражают благодарность Дж. Т. Коул за помощь в подготовке рисунков для данной работы и в работе с пакетом Mathematica. 


\section{Список литературы}

[1] D. J. Korteweg, G. de Vries, "XLI. On the change of form of long waves advancing in a rectangular canal, and on a new type of long stationary waves", Philos. Mag. Ser. 5, 39 (1895), 422-443.

[2] C.S. Gardner, J. M. Greene, M.D. Kruskal, R. M. Miura, "Method for solving the Korteweg-deVries equation", Phys. Rev. Lett., 19:19 (1967), 1095-1097.

[3] P.D. Lax, "Integrals of nonlinear equations of evolution and solitary waves", Commun. Pure Appl. Math., 21:5 (1968), 467-490.

[4] В. Е. Захаров, А. Б. Шабат, "Точная теория двумерной самофокусировки и одномерной автомодуляции волн в нелинейных средах", ЖЖЭТФ, 61:1 (1971), 118-134.

[5] D. J. Benney, A. C. Newell, "The propagation of nonlinear wave envelopes", J. Math. Phys., 46 (1967), 133-139.

[6] M. J. Ablowitz, D. J. Kaup, A. C. Newell, H. Segur, "Nonlinear-evolution equations of physical significance", Phys. Rev. Lett., 31:2 (1973), 125-127.

[7] M. J. Ablowitz, D. J. Kaup, A. C. Newell, H. Segur, "Method for solving the sine-Gordon equation", Phys. Rev. Lett., 30:25 (1973), 1262-1264.

[8] M. J. Ablowitz, D. J. Kaup, A.C. Newell, H. Segur, "The inverse scattering transform-fourier analysis for nonlinear problems", Stud. Appl. Math., 53:4 (1974), 249-315.

[9] М. Абловиц, Х. Сигур, Солитоны и метод обратной задачи, Мир, М., 1987.

[10] В.Е. Захаров, С. В. Манаков, С.П. Новиков, Л.П. Питаевский, Теория солитонов: метод обратной задачи, Наука, М., 1980.

[11] F. Calogero, A. Degasperis, Spectral Transform and Solitons, v. I: Tools to Solve and Investigate Nonlinear Evolution Equations, Lecture Notes in Computer Science, 144, North-Holland, Amsterdam, New York, 1982.

[12] В. Е. Захаров, А. Б. Шабат, "О взаимодействии солитонов в устойчивой среде”, ЖЭЭТФ, 64:5 (1973), 1627-1639.

[13] Л. А. Тахтаджан, Л. Д. Фаддеев, Гамильтонов подход в теории солитонов, Наука, М., 1986.

[14] B. Prinari, M. J. Ablowitz, G. Biondini, "Inverse scattering transform for the vector nonlinear Schrödinger equation with nonvanishing boundary conditions", J. Math. Phys., 47:6 (2006), 063508, 33 pp.

[15] F. Demontis, B. Prinari, C. van der Mee, F. Vitale, "The inverse scattering transform for the defocusing nonlinear Schrödinger equations with nonzero boundary conditions", Stud. Appl. Math., 131:1 (2012), 1-40.

[16] F. Demontis, B. Prinari, C. van der Mee, F. Vitale, "The inverse scattering transform for the focusing nonlinear Schrödinger equations with asymmetric boundary conditions", $J$. Math. Phys., 55:10 (2014), 101505, 40 pp.

[17] G. Biondini, G. Kovačič, "Inverse scattering transform for the focusing nonlinear Schrödinger equation with nonzero boundary conditions", J. Math. Phys., 55:3 (2014), 031506, 22 pp.

[18] G. Biondini, D. Kraus, "Inverse scattering transform for the defocusing Manakov system with nonzero boundary conditions", SIAM J. Math. Anal., 47:1 (2015), 706-757.

[19] G. Biondini, G. Kovačič, D. K. Kraus, "The focusing Manakov system with nonzero boundary conditions", Nonlinearity, 28:9 (2015), 3101-3151.

[20] G. Biondini, D. K. Kraus, B. Prinari, "The three component focusing non-linear Schrödinger equation with nonzero boundary conditions", Commun. Math. Phys., 348:2 (2016), 475-533.

[21] M. Pichler, G. Bondini, "On the focusing non-linear Schrödinger equation with nonzero boundary conditions and double poles", IMA J. Appl. Math., 82:1 (2017), 131-151.

[22] M. J. Ablowitz, Z.H. Musslimani, "Integrable nonlocal nonlinear equations", Stud. Appl. Math., 139:1 (2016), 7-59, arXiv: 1610.02594. 
[23] M. J. Ablowitz, Z.H. Musslimani, "Integrable nonlocal nonlinear Schrödinger equation", Phys. Rev. Lett., 110:6 (2013), 064105, 5 pp.

[24] M. J. Ablowitz, Z. H. Musslimani, "Inverse scattering transform for the integrable nonlocal nonlinear Schrödinger equation", Nonlinearity, 29:3 (2016), 915-946.

[25] A.S. Fokas, "Integrable multidimensional versions of the nonlocal nonlinear Schrödinger equation", Nonlinearity, 29:2 (2016), 319-324.

[26] M. J. Ablowitz, X. D. Luo, Z. H. Musslimani, "The inverse scattering transform for the nonlocal nonlinear Schrödinger equation with nonzero boundary conditions", J. Math. Phys., 59:1 (2018), 011501, 42 pp., arXiv: 1612.02726.

[27] M. J. Ablowitz, B.-F. Feng, X.-D. Luo, Z.H. Musslimani, "Reverse space-time nonlocal sine-Gordon/sinh-Gordon equations with nonzero boundary conditions", Stud. Appl. Math., 2018, Online first DOI: 10.1111/sapm.12222; "Inverse scattering transform for the nonlocal reverse space-time sine-Gordon, sinh-Gordon and nonlinear Schrödinger equations with nonzero boundary conditions", arXiv: 1703.02226. 